\title{
Replacement of fishmeal by yellow mealworm meal on the growth performance, feed utilisation and quality of large yellow croaker
}

\author{
J. Yuan', Y. Wu' ${ }^{1}$ Z.-Y. Zhang1, S.-J. Tian'1, H.-H. Zhou' ${ }^{1}$, W.-B. Zhang ${ }^{1,2^{*}}$ iD and K.-S. Mai ${ }^{1,2}$ \\ ${ }^{1}$ The Key Laboratory of Mariculture (Ministry of Education), The Key Laboratory of Aquaculture Nutrition and Feeds \\ (Ministry of Agriculture and Rural Affairs), Fisheries College, Ocean University of China, Qingdao 266003, China P.R.; \\ ${ }^{2}$ Laboratory for Marine Fisheries Science and Food Production Processes, Qingdao National Laboratory for Marine Science \\ and Technology, Wen Hai Road, Qingdao 266237, China P.R.; wzhang@ouc.edu.cn
}

Received: 21 August 2021 / Accepted: 27 October 2021

(c) 2022 Wageningen Academic Publishers

OPEN ACCESS CC (i) RESEARCH ARTICLE

\begin{abstract}
An 80-day feeding trial was conducted to evaluate the effects of yellow mealworm (Tenebrio molitor, TM) meal as substitute for dietary fishmeal on the growth performance, feed utilisation and flesh quality of large yellow croaker (initial body weight: $189.18 \pm 0.13 \mathrm{~g}$ ). The control diet (TM0) was designed to contain $56 \%$ of fishmeal. Based on the TM0, graded levels of TM meal $(15,30,45,60,75$ and 100\%, respectively) were used to replace fishmeal to formulate the other six experimental diets (TM15, TM30, TM45, TM60, TM75 and TM100), respectively. The results showed that the survival was not significantly affected by dietary TM meal levels $(P>0.05)$. Compared with control group, the final body weight, weight gain rate and protein efficiency ratio decreased significantly when the replacement level over $30 \%$, while feed conversion ratio increased significantly as replacement level over $45 \%(P<0.05)$. The total protein-bound amino acid content in muscle was significantly increased with the increase of dietary TM meal inclusion $(P<0.05)$. With replacement level increasing, the percentage of eicosapentaenoic acid (EPA) and $\sum \mathrm{n}-3 / \sum \mathrm{n}-6$ poly-unsaturated fatty acids (PUFA) in muscle significantly decreased $(P<0.05)$. Meanwhile, the skin redness $\left(\mathrm{a}^{*}\right)$ and yellowness $\left(\mathrm{b}^{*}\right)$ values in the ventral and bottom of ventral regions showed a decreasing and increasing trend, respectively $(P<0.05)$. The TM100 group showed a higher myofibre diameter and lower myofibre density compared to the control group $(P<0.05)$. Total replacement of fishmeal with TM meal significantly down-regulated and up-regulated the expression of myf6 and $m s t n$, respectively $(P<0.05)$. The contents of inosine-5'-monophosphate and total free amino acids were significantly decreased with the increase of TM meal inclusion $(P<0.05)$. In conclusion, TM meal can replace at least $30 \%$ of dietary fishmeal protein without negative effects on the growth, feed utilisation and flesh quality of large yellow croaker.
\end{abstract}

Keywords: large yellow croaker, Tenebrio molitor, fishmeal, growth, quality

\section{Introduction}

The shortage of fishmeal has been the key factor restricting the development of aquaculture industry in recent decades. In order to ensure the sustainable development of aquaculture, finding high quality protein sources to replace dietary fishmeal has always been a hot topic in aquaculture research. Many previous studies in some fish species have shown that some protein sources could partially replace dietary fishmeal without negative effect on growth and feed utilisation, such as soybean meal (gilthead sea bream)
(Martínez-Llorens et al., 2007), cottonseed meal (catfish) (Robinson and Li, 1994), meat and bone meal (Japanese flounder) (Kikuchi et al., 1997) and poultry meal (cobia) (Watson et al., 2014). However, these traditional protein sources still have some shortcomings. For example, the presence of anti-nutritional factors, poor palatability or unbalanced amino acid profile, make them difficult to replace high percentage of fishmeal in aquafeeds (Liu et al., 2020; Sun et al., 2015). Therefore, the research and developing for novel protein sources with better quality to replace fishmeal remain necessary. 
Insects have attracted a lot of attention as novel protein sources used in food and feed fields, due to their excellent nutritional composition and the potential to meet sustainable and accessible principles (Nogales-Mérida et al., 2018; Stamer, 2015; Van Huis, 2020). The yellow mealworm (Tenebrio molitor, TM) belongs to the coleoptera order, Tenebrionidae family. It can convert organic waste into available protein while cause less pollution and consume less resource (Van Huis, 2013; Van Huis and Dunkel, 2017). The TM larva is easy to breed and reproduce, and the products made by them contain high protein level (47-60\%). As a result, the TM meal is widely used in poultry and fish feeds (Gasco et al., 2018; Makkar et al., 2014). Amounts of studies have reported the potential of TM meal as an alternative protein source for fishmeal in some carnivorous mariculture fish. For example, the TM meal could replace 25-50\% dietary fishmeal without negative effect on growth performance of gilthead sea bream (Piccolo et al., 2017), blackspot sea bream (Iaconisi et al., 2017) and rainbow trout (Belforti et al., 2016).

Large yellow croaker (Larimichthys crocea), one of the widely cultured carnivorous fish in China, is favoured by consumers due to its beautiful skin colour and delicious taste. Fishmeal is the main protein source in commercial feed of large yellow croaker. As a result, finding novel protein sources to replace fishmeal is also an urgent problem to be solved in large yellow croaker culture. Although many studies have shown that TM meal could successfully replace part of fishmeal in some carnivorous fish feed, the application of TM meal as substitute for dietary fishmeal for large yellow croaker has not been reported.

With the improvement of living standard, consumers pay more and more attention to the quality of aquaculture products. The quality is a complex concept, mainly including skin and meat colour, condition indices, flesh texture and flavour. It is affected by many factors, such as fish species, age, size, nutritional status, environmental factors and pre- or post-slaughter handling procedures (Bjørnevik et al., 2017; Grigorakis, 2007). The nutritional status is one of the key factors affecting fish quality. At present, studies on fishmeal substitution mainly focus on growth performance and fish health. However, the fish quality affects the acceptance of consumers for farmed fish, especially for large yellow croaker. Thus, the effects of fishmeal replacement by TM meal on quality of large yellow croaker is also worth concerning.

The aim of the present study was to investigate the effects of TM meal as substitute for dietary fishmeal on the growth performance, feed utilisation and flesh quality of large yellow croaker.

\section{Materials and methods}

The present study was carried out strictly according to the recommendations in the Guide for the Use of Experimental Animals of Ocean University of China.

\section{Experimental diets and design}

Seven isonitrogenous (about $46 \%$ of crude protein) and isolipidic (about 9\% of crude lipid) experimental diets were formulated (Table 1). To make floating extruded diets, the dietary starch contents were controlled. The microcrystalline cellulose was used to adjust the experimental diets as isonitrogenous and isolipidic. The control diet used fish meal as main protein source (56\% fish meal) and was named TM0. Based on the control diet, the fishmeal protein was replaced by $15,30,45,60,75$ and $100 \% \mathrm{TM}$ meal protein, respectively, and they were named as TM15, TM30, TM45, TM60, TM75 and TM100, respectively. The amino acid and fatty acid composition of the experimental diets are presented in Table 2 and Table 3.

The experimental diets were made in the Feed Research Institute of Chinese Academy of Agricultural Sciences (Beijing, China). All ingredients were crushed and ground to fine powder, then accurately weighed according to the feed formula and put into a mixer for mixing, and then pellets with a diameter of $6 \mathrm{~mm}$ was extruded by a twin-screw extruder (MY $56 \times 2$, Muyang, Yangzhou, China). After drying, the mixed fish oil, soybean oil and phospholipid oil were evenly sprayed onto the surface of the feed pellets by vacuum coater, and then all diets were packed into small bags and stored at $-20^{\circ} \mathrm{C}$ until use.

\section{Feeding trial}

The feeding trial was carried out in floating sea cages in Ningde, Fujian Province, China. Large yellow croakers were purchased from a commercial hatchery of Fufa (Ningde, Fujian Province, China). Before the formal experiment, the fish were temporarily fed in sea cages $(4.0 \times 8.0 \times 4.0 \mathrm{~m})$ for 2 weeks, during which they were fed with commercial diet to acclimate the feed and environment. At the beginning of the experiment, all fish were starved for 24 hours and weighed. Healthy fish of similar size $(189.18 \pm 0.13 \mathrm{~g})$ were selected and randomly distributed into 21 cages $(2.0 \times 2.0 \times 2.0 \mathrm{~m}, 100$ fish per cage). Each diet was assigned to three cages and the fishes were fed twice daily (5:00 and 18:00, respectively) until apparent satiation. Feeds feeding and fish mortality were recorded daily. During the 80-day feeding trial, the water temperature ranged from 19.8 to $28.5^{\circ} \mathrm{C}$, salinity 31.1 to 34.9 , and the dissolved oxygen content $>6 \mathrm{mg} / \mathrm{l}$. 
Table 1. Formulation and proximate composition of the experimental diets.

\begin{tabular}{|c|c|c|c|c|c|c|c|}
\hline & TMO & TM15 & TM30 & TM45 & TM60 & TM75 & TM100 \\
\hline \multicolumn{8}{|l|}{ Ingredients (\%) } \\
\hline TASA fish meal ${ }^{1}$ & 56.00 & 47.60 & 39.20 & 30.80 & 22.40 & 14.00 & 0.00 \\
\hline Tenebrio molitor meal & 0.00 & 8.52 & 17.05 & 25.57 & 34.1 & 42.62 & 56.83 \\
\hline Tapioca starch & 5.00 & 5.00 & 5.00 & 5.00 & 5.00 & 5.00 & 5.00 \\
\hline Wheat flour & 9.80 & 9.80 & 9.80 & 9.80 & 9.80 & 9.80 & 9.80 \\
\hline Wheat gluten & 5.00 & 5.00 & 5.00 & 5.00 & 5.00 & 5.00 & 5.00 \\
\hline Fish oil & 2.90 & 3.40 & 3.90 & 4.40 & 4.90 & 5.40 & 6.20 \\
\hline Soybean oil & 2.00 & 2.00 & 2.00 & 2.00 & 2.00 & 2.00 & 2.00 \\
\hline Lecithin & 1.50 & 1.50 & 1.50 & 1.50 & 1.50 & 1.50 & 1.50 \\
\hline Premix ${ }^{2}$ & 1.00 & 1.00 & 1.00 & 1.00 & 1.00 & 1.00 & 1.00 \\
\hline Choline chloride & 0.20 & 0.20 & 0.20 & 0.20 & 0.20 & 0.20 & 0.20 \\
\hline Calcium biphosphate & 1.00 & 1.00 & 1.00 & 1.00 & 1.00 & 1.00 & 1.00 \\
\hline Kelp powder & 1.00 & 1.00 & 1.00 & 1.00 & 1.00 & 1.00 & 1.00 \\
\hline Microcrystalline cellulose & 14.20 & 13.58 & 12.95 & 12.33 & 11.70 & 11.08 & 10.07 \\
\hline Glycine & 0.10 & 0.10 & 0.10 & 0.10 & 0.10 & 0.10 & 0.10 \\
\hline Betaine & 0.20 & 0.20 & 0.20 & 0.20 & 0.20 & 0.20 & 0.20 \\
\hline $\mathrm{Y}_{2} \mathrm{O}_{3}$ & 0.10 & 0.10 & 0.10 & 0.10 & 0.10 & 0.10 & 0.10 \\
\hline Total & 100.00 & 100.00 & 100.00 & 100.00 & 100.00 & 100.00 & 100.00 \\
\hline \multicolumn{8}{|c|}{ Proximate composition ( $\%$, dry matter) } \\
\hline Dry matter & 95.75 & 95.79 & 95.90 & 96.29 & 95.57 & 95.54 & 95.52 \\
\hline Crude lipid & 9.25 & 9.58 & 9.08 & 9.04 & 9.10 & 9.23 & 9.13 \\
\hline Crude protein & 45.68 & 46.27 & 46.29 & 46.34 & 46.73 & 46.14 & 46.39 \\
\hline Ash & 12.67 & 11.37 & 11.24 & 10.32 & 9.88 & 8.67 & 7.30 \\
\hline Gross energy (MJ/kg) & 20.25 & 20.86 & 20.53 & 21.05 & 20.75 & 21.24 & 20.57 \\
\hline
\end{tabular}

\section{Sample collection}

At the end of the feeding trial, the fish were fasted for 24 hours and anesthetised with eugenol (1:10,000) (purity 99\%, Shanghai Reagent, Shanghai, China). The fish were counted and weighted to calculate the survival (SR) and weight gain rate (WGR). Ten fish per cage were randomly sampled to measure body length and weight of body, viscera and liver for condition indices. Blood samples were collected from the caudal vein and allowed to clot for $4 \mathrm{~h}$ at $4{ }^{\circ} \mathrm{C}$. The serum was separated by centrifugation $(3,000 \mathrm{rpm} / \mathrm{min}$, $10 \mathrm{~min}, 4{ }^{\circ} \mathrm{C}$ ) and stored at $-80{ }^{\circ} \mathrm{C}$ until use. The dorsal muscle and intestine samples were collected immediately frozen in liquid nitrogen and then stored at $-80{ }^{\circ} \mathrm{C}$ until use. The dorsal muscle of another three fish in each cage were carefully sampled and fixed in $10 \%$ formaldehyde for histological analysis.

Another four fish from each cage were placed on ice and immediately delivered to the laboratory. Dorsal fillet above epaxial myotomes and below dorsal fin of one side was sampled for analysis of $\mathrm{pH}$, drip loss, cooking loss, texture parameters and volatile compounds within 24 hours.

According to the method of Yi et al. (2014), six fish were randomly selected from each cage to measure the skin colour of the dorsal, ventral and caudal regions during 20:00 to 23:00 at night. A portable Minolta Chroma Meter CR-400 (Minolta, Osaka, Japan) was used. According to the recommendation of International Commission on Illumination (CIE, 1976), the skin colour parameters are represented by $\mathrm{L}^{*}$, a* and $\mathrm{b}^{*}$ respectively for brightness, redness and yellowness.

\section{Experimental diets and fish muscle composition analysis}

The approximate composition of experimental diets and fish muscle were determined referring to AOAC standard method (AOAC, 1995). Moisture was determined by drying diets and muscle samples at $105^{\circ} \mathrm{C}$ to constant 
Table 2. Amino acid composition of the experimental diets (\%, dry matter). ${ }^{1}$

\begin{tabular}{|c|c|c|c|c|c|c|c|}
\hline & \multicolumn{7}{|l|}{ Diets } \\
\hline & TMO & TM15 & TM30 & TM45 & TM60 & TM75 & TM100 \\
\hline Arginine & 2.58 & 2.64 & 2.56 & 2.63 & 2.59 & 2.63 & 2.61 \\
\hline Histidine & 1.26 & 1.11 & 1.08 & 0.99 & 0.90 & 0.71 & 0.62 \\
\hline Isoleucine & 1.81 & 2.00 & 1.88 & 1.95 & 1.92 & 1.94 & 1.92 \\
\hline Leucine & 3.18 & 3.33 & 3.22 & 3.38 & 3.33 & 3.34 & 3.33 \\
\hline Lysine & 3.03 & 3.08 & 2.98 & 3.06 & 2.96 & 2.92 & 2.86 \\
\hline Methionine & 0.96 & 1.12 & 1.03 & 1.04 & 0.96 & 0.92 & 0.89 \\
\hline Phenylalanine & 2.30 & 2.10 & 2.10 & 2.11 & 2.09 & 2.09 & 2.14 \\
\hline Threonine & 1.80 & 1.84 & 1.71 & 1.80 & 1.70 & 1.74 & 1.70 \\
\hline Valine & 2.18 & 2.43 & 2.36 & 2.52 & 2.56 & 2.66 & 2.72 \\
\hline EAA & 19.10 & 19.65 & 18.92 & 19.48 & 19.01 & 18.95 & 18.79 \\
\hline Alanine & 2.76 & 3.01 & 3.13 & 3.29 & 3.46 & 3.58 & 3.80 \\
\hline Aspartic acid & 3.20 & 3.58 & 3.33 & 3.31 & 3.19 & 3.32 & 3.17 \\
\hline Cysteine & 0.32 & 0.35 & 0.34 & 0.36 & 0.38 & 0.39 & 0.47 \\
\hline Glutamic acid & 6.87 & 6.88 & 6.68 & 6.89 & 6.62 & 6.49 & 6.30 \\
\hline Glycine & 2.23 & 2.18 & 2.06 & 2.08 & 1.96 & 1.86 & 1.74 \\
\hline Proline & 1.90 & 1.89 & 2.07 & 2.35 & 2.40 & 2.35 & 2.45 \\
\hline Serine & 1.67 & 2.04 & 2.20 & 2.54 & 2.78 & 3.01 & 3.39 \\
\hline Tyrosine & 1.31 & 1.47 & 1.40 & 1.47 & 1.46 & 1.49 & 1.52 \\
\hline NEAA & 20.26 & 21.40 & 21.21 & 22.29 & 22.25 & 22.49 & 22.84 \\
\hline
\end{tabular}

${ }^{1} \mathrm{EAA}=$ essential amino acids; NEAA = non-essential amino acids.

weight. Crude protein was determined using the Kjeldahl (2300-Kjeldahl apparatus, FOSS, Hillerød Denmark) method by measuring nitrogen $(\mathrm{N} \times 6.25)$. The Soxhlet method (Soxhlet extraction system B-811, FOSS) was used to measure crude lipid. Ash was determined by combustion. The sample was placed in muffle furnace and burned at $550{ }^{\circ} \mathrm{C}$ about $4 \mathrm{~h}$ to constant weight. Gross energy was determined using an Oxygen Bomb Calorimeter (Parr 6400, Parr Instrument Company, Moline, IL, USA).

Muscle samples used to determine protein-bound amino acids were freeze-dried and $30 \mathrm{mg}$ of each sample was hydrolysed in $15 \mathrm{ml} 6 \mathrm{~N} \mathrm{HCl}$ solution at $110{ }^{\circ} \mathrm{C}$ for $24 \mathrm{~h}$. Amino acid profile were determined by automatic amino acid analyser (L-8900, Hitachi, Tokyo, Japan).

The analysis of free amino acids was carried out using the method of Wei et al. (2019b). One gram of muscle sample was homogenised with $3 \mathrm{ml} 10 \%$ sulfosalicylic acid for $1 \mathrm{~min}$. After centrifugation, the supernatant was extracted and filtered into the sample bottle for analysis using automatic amino acid analyser (L-8900, Hitachi).

For the analysis of fatty acids, the sample pretreatment method was referred to by Ma et al. (2019). Fatty acids were
Table 3. Fatty acid composition of the experimental diets (\% total fatty acids). ${ }^{1}$

\begin{tabular}{|c|c|c|c|c|c|c|c|}
\hline & \multicolumn{7}{|l|}{ Diets } \\
\hline & TMO & TM15 & TM30 & TM45 & TM60 & TM75 & TM100 \\
\hline $\mathrm{C} 14: 0$ & 5.87 & 5.88 & 5.78 & 5.01 & 4.45 & 4.28 & 3.30 \\
\hline C16:0 & 42.09 & 41.40 & 40.11 & 40.06 & 39.40 & 38.12 & 36.43 \\
\hline C18:0 & 12.31 & 12.30 & 11.96 & 13.33 & 14.53 & 13.85 & 15.05 \\
\hline $\mathrm{C} 20: 0$ & 0.53 & 0.69 & 0.76 & 1.05 & 1.35 & 1.23 & 1.60 \\
\hline C16: n-7 & 2.74 & 2.45 & 2.48 & 2.36 & 2.05 & 1.94 & 1.38 \\
\hline C18: n-9 & 4.10 & 3.68 & 3.77 & 3.95 & 4.03 & 4.24 & 4.28 \\
\hline C18:2n-6 & 16.90 & 19.06 & 20.67 & 20.97 & 21.86 & 24.16 & 26.98 \\
\hline C20: n-9 & 0.90 & 0.86 & 0.86 & 0.95 & 1.04 & 0.93 & 1.08 \\
\hline C18:3n-3 & 2.28 & 2.43 & 2.89 & 2.82 & 2.88 & 3.45 & 3.78 \\
\hline C20:4n-6 & 0.29 & 0.26 & 0.26 & 0.22 & 0.19 & 0.17 & .11 \\
\hline C20:5n-3(EPA) & 4.39 & 3.95 & 3.94 & 3.12 & 2.55 & 2.37 & 1.48 \\
\hline C22:6n-3(DHA) & 4.65 & 4.10 & 3.98 & 3.23 & 2.68 & 2.37 & 1.52 \\
\hline Other $\mathrm{FA}^{2}$ & 2.94 & 2.97 & 2.80 & 2.93 & 2.99 & 2.89 & 3.02 \\
\hline$\sum$ SFA & 62.95 & 62.45 & 60.44 & 61.60 & 61.95 & 59.56 & 58.66 \\
\hline$\sum$ MUFA & 8.06 & 7.28 & 7.31 & 7.50 & 7.40 & 7.34 & 6.96 \\
\hline$\sum$ PUFA & 28.99 & 30.28 & 32.25 & 30.90 & 30.65 & 33.10 & 34.39 \\
\hline$\sum n-3$ PUFA & 11.38 & 11.15 & 10.87 & 9.23 & 8.20 & 8.28 & 6.87 \\
\hline$\sum n-6$ PUFA & 17.61 & 19.74 & 21.38 & 21.67 & 22.45 & 24.82 & 27.52 \\
\hline$\sum n-3 / \sum n-6$ PUFA & 0.65 & 0.54 & 0.51 & 0.43 & 0.37 & 0.33 & 0.25 \\
\hline $\begin{array}{l}\text { = mono-unsaturate } \\
\text { acids; PUFA = poly } \\
\text { acids. } \\
2 \text { Other fatty acid: } \\
\text { C22:0, C20:3n-6, } \\
\text { C24:n-9 were also } \\
\text { They were utilised }\end{array}$ & $\begin{array}{l}\text { d fatty a } \\
\text {-unsatur } \\
\text { C15:0, C } \\
22: n-9 \text {, } \\
\text { detectec }\end{array}$ & $\begin{array}{l}\text { acids; } n- \\
\text { urated fa } \\
\text { C17:0, C } \\
9, \mathrm{C} 20: 3 \mathrm{r} \\
\text { d but no } \\
\text { ulate the }\end{array}$ & $\begin{array}{l}\text { C17:n-7, } \\
\text { 3n-3, C2 } \\
\text { ot repor } \\
\text { e fatty a }\end{array}$ & $\begin{array}{l}\text { C18:3 } \\
23: 0, C 2 \\
\text { rted in th } \\
\text { icid grou }\end{array}$ & $\begin{array}{l}\text { cids; } n-6 \\
=\text { satura } \\
n-6, \text { c21 } \\
22: 2 n-6, \\
\text { he table } \\
\text { ups. }\end{array}$ & $\begin{array}{l}1: 0, C 20 \\
C 24: 0 \text { a } \\
\text { for low }\end{array}$ & $\begin{array}{l}\text { MUFA } \\
\text { fatty } \\
\text { ty } \\
0: 2 n-6 \text {, } \\
\text { and } \\
\text { levels. }\end{array}$ \\
\hline
\end{tabular}

determined by gas chromatography-mass spectrometry (GCMS-QP2010, Shimadzu, Kyoto, Japan) fitted with an automatic sampler. Fatty acids were identified based on a mass spectrometry database retrieval (similarity $>80 \%$ ), and the results were expressed as the percentage of each fatty acid in total fatty acids.

\section{Biochemical indexes assay in intestine}

The fish intestine was divided into anterior intestine, midintestine and posterior intestine during sampling, and the midgut was taken for enzyme activity determination. The midgut of each group was weighed and homogenised into $0.01 \mathrm{M}$ buffer solution. The activities of amylase (AMS), lipase (LPS), trypsin, chymotrypsin, creatine kinase (CK), $\gamma$-glutamyltransferase $\left(\gamma\right.$-GT), $\mathrm{Na}^{+} \mathrm{K}^{+}$-ATPase and alkaline phosphatase (AKP) were measured by using commercial kits (Nanjing Jiancheng Bioengineering Institute, Nanjing, 
China), and they were AMS (C016-1-1), LPS (A054-2-1), trypsin (A080-2-2), chymotrypsin (A080-3-1), CK (A0321-1), $\gamma$-GT (C017-2-1), $\mathrm{Na}^{+} \mathrm{K}^{+}$-ATPase (A070-2-2) and AKP (A059-2-2), respectively. All procedures were carried out according to the instructions of the commercial kits.

\section{Muscle $\mathrm{pH}$, water holding capacity and texture analysis}

Muscle $\mathrm{pH}$ value was determined using a digital display $\mathrm{pH}$ meter according to the method of Fuentes et al. (2010). The muscle $(\mathrm{g}) /$ distilled water $(\mathrm{ml})=1: 9$ was homogenised and measured with $\mathrm{pH}$ meter (PB-10, Sartorius, Göttingen, Germany).

Water holding capacity (WHC) was expressed by cooking loss and drip loss. The analysis of WHC was performed with previous studies with minor modification (Lv et al., 2021; Sánchez-Alonso et al., 2007). Fish fillet $(5 \times 3 \times 1$ $\mathrm{cm})$ was cut from left side of the fish, weighted $\left(\mathrm{W}_{1}\right)$ and suspended in a plastic bag, where small holes had been made to drain the drip. Samples were placed at $2-4{ }^{\circ} \mathrm{C}$ for $48 \mathrm{~h}$ and weighed again $\left(\mathrm{W}_{2}\right)$ to determine the drip loss. Samples of the same size were weighed and placed in retort pouch then cooked in a water bath at $100{ }^{\circ} \mathrm{C}$ for 15 minutes. The fish fillets were taken out and the surface water were dried by paper towels, then reweighed to calculate the cooking loss according to the following equation:

Drip loss $(\%)=100 \times \frac{W_{1}-W_{2}}{W_{1}}$

Cooking loss $(\%)=100 \times \frac{\text { the weight before cooking }- \text { the weight after cooking }}{\text { the weight before cooking }}$

Texture (hardness, cohesiveness, adhesiveness, springiness, chewiness and gumminess) analysis was performed by a texture analyser (TMS-TOUCH, Food Technology Corporation, West Sussex, VA, USA) with the method of Ginés et al. (2004). The muscle above the lateral line $(1.5 \times 1.5 \times 1.0 \mathrm{~cm})$ of fish was used for texture analysis. An 8 $\mathrm{mm}$ cylinder probe and double compression was applied to construct the texture profile analyses parameters. The test condition involved two consecutive cycles of compression with a constant speed of $30 \mathrm{~mm} / \mathrm{min}$ with the deformation $60 \%$ of the original length, and the initial force was $0.1 \mathrm{~N}$ (Wei et al., 2016). The shear force was determined by the combination single blade and meat shear cell.

\section{Muscle hydroxyproline, collagen, water and salt soluble protein determination}

The content of hydroxyproline was determined using the commercial kit (A030-2-1, Nanjing Jiancheng Bioengineering Institute, Nanjing, China). The collagen content was estimated by multiplying the hydroxyproline content by 8 (AOAC, 2000).
Water-soluble protein and salt-soluble protein were extracted with phosphate buffer (0.05 M, pH 7.0) alone or with $0.6 \mathrm{M} \mathrm{KCl}$ (Sigholt et al., 1997), and the protein content was determined using the Coomassie brilliant blue method of TP commercial kit (A045-2-2, Nanjing Jiancheng Bioengineering Institute, Nanjing, China).

\section{Muscle nucleotides content and freshness assay}

The nucleotide content was analysed by HPLC (LC20AT, Shimadzu) with the method of Wei et al. (2019a), including inosine-5'-monophosphate (IMP), adenosine5 '-triphosphate (ATP), adenosine-5'-diphosphate (ADP), adenosine-5'-monophosphate (AMP), inosine (Ino) and hypoxanthine $(\mathrm{Hx})$. The $\mathrm{K}$ and $\mathrm{Ki}$ values were used to evaluate muscle freshness, and the calculation equations are as follows:

$$
\begin{aligned}
& K(\%)=100 \times \frac{\text { Ino }+ \text { Hx }}{\text { ATP }+ \text { ADP }+ \text { AMP }+ \text { IMP }+ \text { Ino }+ \text { Hx }} \\
& K i(\%)=100 \times \frac{\text { Ino }+ \text { Hx }}{\text { IMP + Ino + Hx }}
\end{aligned}
$$

\section{Volatile compounds analysis}

The content of volatile compounds in muscle was determined by gas chromatograph and ion mobility spectrometry (FlavourSpec ${ }^{\circ}$, G.A.S., Dortmund, Germany) (GC-IMS) equipped with an automatic sampling device. Briefly, muscle sample $(3 \mathrm{~g})$ was transferred into a $20 \mathrm{ml}$ headspace bottle carefully and then incubated at $55^{\circ} \mathrm{C}$, while being stirred at $250 \mathrm{rpm}$ for $15 \mathrm{~min}$. After incubation, $500 \mathrm{ul} \mathrm{headspace}$ was injected using an $85^{\circ} \mathrm{C}$ heated syringe into a FS-SE-54 capillary column in splitless mode. Pure nitrogen was used as the carrier gas with programmed flow: $2 \mathrm{ml} / \mathrm{min}$ for $2 \mathrm{~min}$, ramp to $100 \mathrm{ml} / \mathrm{min}$ over $20 \mathrm{~min}$, and maintained for $10 \mathrm{~min}$ until stopping. Analytes were driven to the ionisation chamber by a $3 \mathrm{H}$ ionisation source in positive ion mode. The $9.8 \mathrm{~cm}$ drift tube was operated at $45^{\circ} \mathrm{C}$ with $150 \mathrm{ml} / \mathrm{min}$ nitrogen flow. Volatile compounds identification was mainly based on the comparison of retention index and drift time in GC-IMS library database.

\section{Histology analysis}

The sections were stained with picrosirius red using the slide stainer and observed under light microscope. Meanwhile, the micrograph of each group was taken and the muscle fibre density and diameter were calculated.

\section{Gene expression in muscle}

Total RNA was isolated from muscle using RNAiso Plus Kit (9109, Takara, Japan). The quality of RNA was detected by agarose gel electrophoresis at $1.2 \%$, the concentration of RNA was assessed by Nano-Drop 2000 spectrophotometer (Thermo Fisher Scientific, Waltham, MA, USA). The cDNA 
was generated by using PrimeScript RT reagent Kit with gDNA Eraser (RR047A, Takara, Kusatsu, Japan) following the manufacturer's protocol. The mRNA levels of myogenic differentiation $(M y o D)$, myogenin $(M y o G)$, myogenic factor 5 (myf5), myogenic factor 6 (myf6), paired box 7 (Pax-7) and myostatin (mstn) were analysed by Real-time PCR system (Quant Studio 5, Applied Biosystems, Waltham, MA, USA). And $\beta$-actin was used as the internal reference to normalise the mRNA expression level of the control group. The results of gene expression were analysed according to 2- $\triangle \Delta C T$ method. The primers used for the real-time PCR analysis are shown in Table 4.

\section{Calculations and statistical analysis}

The survival, growth performance, feed utilisation and body condition indices of large yellow croaker were calculated as follows:

$$
\begin{aligned}
& \text { Survival }(\mathrm{SR}, \%)=100 \times \frac{\text { Final fish number }}{\text { Initial fish number }} \\
& \text { Weight gain rate }(\mathrm{WGR}, \%)=100 \times \frac{\text { final body weight }- \text { initial body weight }}{\text { initial body weight }} \\
& \text { Protein efficiency ratio }(\mathrm{PER}, \%)=100 \times \frac{\text { Weight gain }(\mathrm{g})}{\text { Total protein fed }(\mathrm{g} \text {, dry basis })} \\
& \text { Feed conversion ratio }(\mathrm{FCR})=\frac{\text { Total feed intake }(\mathrm{g} \text {, dry basis })}{\text { Weight gain }(\mathrm{g})} \\
& \text { Feed intake }(\mathrm{FI}, \% / \text { day })= \\
& 100 \times \frac{\text { Total feed intake }(\mathrm{g} \text {, dry basis })}{\text { Number of feeding days } \times \frac{\text { final body weight }+ \text { initial body weight }}{2}} \\
& \text { Hepatosomatic index }(\mathrm{HSI}, \%)=100 \times \frac{\text { Hepatic weight }}{\text { Body weight }} \\
& \text { Viscerosomatic index }(\mathrm{VSI}, \%)=100 \times \frac{\text { Viscera weight }}{\text { Body weight }} \\
& \text { Condition factor }(\mathrm{CF})=100 \times \frac{\text { Body weight }}{\text { Total length } \left.(\mathrm{cm})^{3}\right)}
\end{aligned}
$$

All experimental data were analysed using the software of SPSS 25.0 (IBM Corp., New York, NY, USA). One-way analysis of variance (ANOVA) followed by Tukey's multiple range test was used to determine whether different levels of substitution had significant effects on the measured indicators. The significance level was set as $P<0.05$.
All experimental results were presented as means \pm SE (standard error of the mean).

\section{Results}

\section{Growth performance and body condition indices}

The data on growth performance and condition indices are shown in Table 5. There was no significant difference in survival (84.67-94.67\%) among all the treatments $(P>0.05)$. Compared with control group, the finial body weight, WGR and protein efficiency ratio significantly decreased when the level of replacement over $30 \%(P<0.05)$, while the FCR significantly increased as the proportion of substitution over $45 \%(P<0.05)$. The FI in TM75 and TM100 groups were significantly lower than those in the other groups $(P<0.05)$. The TM100 group had significantly lower viscerosomatic index and hepatosomatic index than those in the other groups $(P<0.05)$. The CF in TM60, TM75 and TM100 groups were significantly decreased compared with those in the other groups $(P<0.05)$.

\section{Muscle composition}

The data on muscle composition are shown in Table 6. There was no significant difference in muscle moisture among the treatments $(P>0.05)$. The contents of crude protein and ash in TM100 group were significantly higher than that in the control group $(P<0.05)$, while crude lipid content showed an opposite trend. The contents of muscle hydroxyproline and collagen in the TM75 and TM100 groups were significantly lower than that in the control group $(P<0.05)$. Water soluble protein content was not significantly affected by dietary composition $(P>0.05)$. But a reduction of salt soluble protein content was found with the dietary inclusion of TM meal increasing $(P<0.05)$.

\section{Muscle protein-bound amino acid and fatty acid}

According to Table 7, the essential amino acid (EAA) and non-essential amino acid (NEAA) contents significantly increased when the replacement level up to $45 \%(P<0.05)$.

Table 4. The primers used for the real-time PCR analysis.

$\begin{array}{llll}\text { Gene } & \text { Forward (5'-3') } & \text { Reverse (5'-3') } & \text { GenBank accession no. } \\ \text { MyoD } & \text { ACAGCAGCTCTTATTTCTCCGA } & \text { GTCATTCTTCAGACCGCCGTT } & \text { XM_010745476.3 } \\ \text { MyoG } & \text { GGAGCTTTTCGAGACCAACCC } & \text { AGATTCCCACACAAGCCCAT } & \text { XM_010738811.3 } \\ \text { myf5 } & \text { CAACTGCTCTGACGGCAT } & \text { CGCACAGACTCTCATTCTTCG } & \text { XM_019276871.2 } \\ \text { myf6 } & \text { CCGAACCAGAGGCTACCCAA } & \text { TAACCGCTCGATGTAGCTGA } & \text { XM_010737255.3 } \\ \text { Pax-7 } & \text { ACCACCTTCACCGCTGAG } & \text { CTCGCCTGTTGCTAAACCAC } & \text { XM_010731346.3 } \\ \text { mstn } & \text { GTCGCCCATCAACATGCTCT } & \text { GAGCATCCACAACGGTCCAC } & \text { XM_010733150.3 } \\ \text { B-actin } & \text { GACCTGACAGACTACCTCATG } & \text { AGTTGAAGGTGTCTCGTGGA } & \text { GU584189 }\end{array}$


Table 5. Effects of fishmeal replacement by TM meal on the growth performance and body condition indices of large yellow croaker. ${ }^{1,2}$

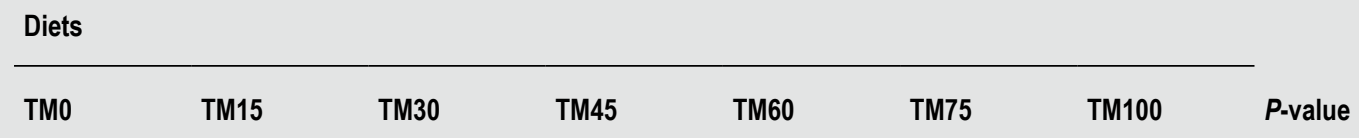

Growth performance

$\begin{array}{lcccccccc}\text { SR (\%) } & 91.00 \pm 5.03 & 94.00 \pm 2.52 & 94.67 \pm 1.67 & 89.33 \pm 1.76 & 91.00 \pm 1.53 & 84.67 \pm 5.90 & 87.33 \pm 3.33 & 0.45 \\ \text { IBW(g) } & 189.27 \pm 0.27 & 189.47 \pm 0.13 & 189.93 \pm 0.35 & 188.73 \pm 0.29 & 188.93 \pm 0.07 & 188.73 \pm 0.35 & 189.2 \pm 0.50 & 0.15 \\ \text { FBW(g) } & 322.94 \pm 14.34^{\mathrm{a}} & 319.06 \pm 7.90^{\mathrm{a}} & 322.91 \pm 6.34^{\mathrm{a}} & 284.67 \pm 3.91^{\mathrm{b}} & 277.78 \pm 3.05^{\mathrm{b}} & 230.28 \pm 2.07^{\mathrm{c}} & 209.66 \pm 0.75^{\mathrm{c}} & 0.00 \\ \text { WGR (\%) } & 70.62 \pm 7.55^{\mathrm{a}} & 68.40 \pm 4.29^{\mathrm{ab}} & 70.02 \pm 3.57^{\mathrm{a}} & 50.82 \pm 1.84^{\mathrm{bc}} & 47.03 \pm 1.66^{\mathrm{c}} & 22.02 \pm 1.27^{\mathrm{d}} & 10.82 \pm 0.61^{\mathrm{d}} & 0.00 \\ \text { FCR } & 1.48 \pm 0.03^{\mathrm{d}} & 1.52 \pm 0.11^{\mathrm{d}} & 1.46 \pm 0.06^{\mathrm{d}} & 1.94 \pm 0.02^{\mathrm{cd}} & 2.00 \pm 0.06^{\mathrm{c}} & 2.93 \pm 0.11^{\mathrm{b}} & 4.31 \pm 0.20^{\mathrm{a}} & 0.00 \\ \text { PER (\%) } & 1.34 \pm 0.08^{\mathrm{a}} & 1.36 \pm 0.06^{\mathrm{a}} & 1.41 \pm 0.06^{\mathrm{a}} & 1.00 \pm 0.01^{\mathrm{b}} & 0.99 \pm 0.02^{\mathrm{b}} & 0.63 \pm 0.06^{\mathrm{c}} & 0.44 \pm 0.03^{\mathrm{c}} & 0.00 \\ \text { FI (\%/d) } & 0.96 \pm 0.07^{\mathrm{a}} & 0.96 \pm 0.03^{\mathrm{a}} & 0.94 \pm 0.01^{\mathrm{a}} & 0.98 \pm 0.04^{\mathrm{a}} & 0.96 \pm 0.05^{\mathrm{a}} & 0.72 \pm 0.01^{\mathrm{b}} & 0.55 \pm 0.01^{\mathrm{b}} & 0.00 \\ \text { Body condition indices } & & & & & & & & \\ \text { VSI (\%) } & 4.97 \pm 0.15^{\mathrm{a}} & 5.13 \pm 0.14^{\mathrm{a}} & 5.10 \pm 0.14^{\mathrm{a}} & 5.10 \pm 0.14^{\mathrm{a}} & 5.12 \pm 0.19^{\mathrm{a}} & 5.03 \pm 0.14^{\mathrm{a}} & 4.51 \pm 0.14^{\mathrm{b}} & 0.04 \\ \text { HSI (\%) } & 2.75 \pm 0.13^{\mathrm{a}} & 2.89 \pm 0.11^{\mathrm{a}} & 2.76 \pm 0.11^{\mathrm{a}} & 3.06 \pm 0.13^{\mathrm{a}} & 2.80 \pm 0.13^{\mathrm{a}} & 2.64 \pm 0.11^{\mathrm{a}} & 1.94 \pm 0.09^{\mathrm{b}} & 0.00 \\ \text { CF } & 1.83 \pm 0.02^{\mathrm{a}} & 1.81 \pm 0.02^{\mathrm{a}} & 1.80 \pm 0.01^{\mathrm{a}} & 1.76 \pm 0.02^{\mathrm{ab}} & 1.71 \pm 0.02^{\mathrm{b}} & 1.58 \pm 0.01^{\mathrm{c}} & 1.49 \pm 0.01^{\mathrm{d}} & 0.00\end{array}$

${ }^{1} \mathrm{CF}=$ condition factor; FBW = finial body weight; FCR = feed conversion ratio; FI = feed intake; $\mathrm{HSI}=$ hepatosomatic index; IBW = initial body weight; $\mathrm{PER}=$ protein efficiency ratio; $\mathrm{SR}=$ survival rate; $\mathrm{VSI}=$ viscerosomatic index; $\mathrm{WGR}=$ weight gain rate.

${ }^{2}$ Values are mean \pm SE. Mean values within the same row with different superscripts are significantly different $(P<0.05)$.

Table 6. Effects of fishmeal replacement by TM meal on dorsal muscle composition of large yellow croaker (wet weight). ${ }^{1}$

\begin{tabular}{|c|c|c|c|c|c|c|c|c|}
\hline & \multicolumn{7}{|l|}{ Diets } & \multirow[b]{2}{*}{$P$-value } \\
\hline & TMO & TM15 & TM30 & TM45 & TM60 & TM75 & TM100 & \\
\hline Moisture (\%) & $69.68 \pm 1.05$ & $68.16 \pm 1.16$ & $70.62 \pm 0.72$ & $70.32 \pm 0.79$ & $69.54 \pm 0.12$ & $70.12 \pm 0.55$ & $71.24 \pm 0.57$ & 0.16 \\
\hline Crude protein (\%) & $16.32 \pm 0.06^{\mathrm{bc}}$ & $16.63 \pm 0.05^{a b c}$ & $15.88 \pm 0.24^{c}$ & $16.31 \pm 0.23^{b c}$ & $16.83 \pm 0.14^{\mathrm{ab}}$ & $16.78 \pm 0.27^{a b}$ & $17.29 \pm 0.16^{a}$ & 0.00 \\
\hline Crude lipid (\%) & $12.63 \pm 0.11^{a}$ & $13.37 \pm 0.13^{a}$ & $12.94 \pm 0.45^{a}$ & $12.94 \pm 0.24^{\mathrm{a}}$ & $12.84 \pm 0.09^{a}$ & $12.6 \pm 0.06^{\mathrm{ab}}$ & $11.64 \pm 0.22^{b}$ & 0.00 \\
\hline Ash (\%) & $3.38 \pm 0.11^{b}$ & $3.21 \pm 0.06^{b}$ & $3.24 \pm 0.10^{b}$ & $3.25 \pm 0.17^{b}$ & $3.34 \pm 0.08^{b}$ & $3.58 \pm 0.09^{\mathrm{ab}}$ & $3.88 \pm 0.06^{a}$ & 0.00 \\
\hline Hydroxyproline (mg/g) & $0.31 \pm 0.01^{\mathrm{a}}$ & $0.30 \pm 0.01^{\mathrm{a}}$ & $0.31 \pm 0.04^{a}$ & $0.26 \pm 0.01^{\mathrm{ab}}$ & $0.25 \pm 0.01^{\mathrm{ab}}$ & $0.22 \pm 0.02^{b}$ & $0.22 \pm 0.03^{b}$ & 0.02 \\
\hline Collagen (mg/g) & $2.45 \pm 0.03^{\mathrm{a}}$ & $2.40 \pm 0.05^{\mathrm{a}}$ & $2.52 \pm 0.28^{a}$ & $2.07 \pm 0.01^{\mathrm{ab}}$ & $2.00 \pm 0.01^{\mathrm{ab}}$ & $1.79 \pm 0.19^{b}$ & $1.79 \pm 0.25^{b}$ & 0.02 \\
\hline Salt soluble protein $(\mathrm{g} / 100 \mathrm{~g})$ & $6.85 \pm 0.02^{\mathrm{a}}$ & $6.45 \pm 0.16^{\mathrm{a}}$ & $5.58 \pm 0.23^{b c}$ & $5.92 \pm 0.11^{b}$ & $5.20 \pm 0.15^{\mathrm{cd}}$ & $5.41 \pm 0.10^{\mathrm{cd}}$ & $5.03 \pm 0.06^{d}$ & 0.00 \\
\hline Water soluble protein $(\mathrm{g} / 100 \mathrm{~g})$ & $4.36 \pm 0.18$ & $4.18 \pm 0.22$ & $3.98 \pm 0.07$ & $3.91 \pm 0.07$ & $3.91 \pm 0.07$ & $3.97 \pm 0.06$ & $3.92 \pm 0.06$ & 0.11 \\
\hline
\end{tabular}

${ }^{1}$ Values are mean \pm SE. Mean values within the same row with different superscripts are significantly different $(P<0.05)$.

However, the ratio of EAA/NEAA was not significantly affected by dietary TM meal levels $(P>0.05)$.

According to Table 8, no significant difference was observed in docosahexaenoic acid (DHA) (C22:6n-3), saturated fatty acids, mono-unsaturated fatty acids and PUFA among all the treatments $(P>0.05)$. The concentration of EPA (C20:5n-3) and $\Sigma$ n-3 PUFA were significantly decreased following the increase of TM meal inclusion $(P<0.05)$. However, the percentage of Linoleic acid (C18:2n-6) and $\Sigma$ n-6 PUFA were significantly increased with increasing dietary TM meal levels $(P<0.05)$. Therefore, this led to a significant reduction of the $\sum \mathrm{n}-3 / \sum \mathrm{n}-6$ PUFA ratio $(P<0.05)$.

\section{Enzyme activities in intestine}

Results of intestinal enzyme activities are presented in Table 9. Digestive enzymes activity such as AMS, LPS, trypsin and chymotrypsin, were significantly decreased when the level of substitution over $45 \%(P<0.05)$. As for absorptive enzymes, the activities of $\mathrm{CK}$ and $\gamma$-GT significantly decreased as the dietary TM level increased above $30 \%$ $(P<0.05)$, the AKP activity significantly decreased when the substitution level up to $60 \%(P<0.05)$. And $\mathrm{Na}^{+} \mathrm{K}^{+}$-ATPase activity was significantly lower in the TM75 and TM100 groups than that in the control group $(P<0.05)$. 
Table 7. Effects of fishmeal replacement by TM meal on protein-bound amino acid composition of large yellow croaker (\%, dry matter). ${ }^{1,2}$

\begin{tabular}{|c|c|c|c|c|c|c|c|c|}
\hline & \multicolumn{7}{|l|}{ Diets } & \multirow[b]{2}{*}{$P$-value } \\
\hline & TMO & TM15 & TM30 & TM45 & TM60 & TM75 & TM100 & \\
\hline \multicolumn{9}{|l|}{ EAA } \\
\hline Arginine & $3.32 \pm 0.17^{b}$ & $3.29 \pm 0.01^{b}$ & $3.28 \pm 0.02^{b}$ & $3.27 \pm 0.09^{b}$ & $3.57 \pm 0.01^{b}$ & $3.48 \pm 0.07^{b}$ & $4.04 \pm 0.10^{\mathrm{a}}$ & 0.00 \\
\hline Histidine & $1.16 \pm 0.05^{b}$ & $1.19 \pm 0.05^{\mathrm{ab}}$ & $1.29 \pm 0.03^{\mathrm{ab}}$ & $1.37 \pm 0.07^{\mathrm{ab}}$ & $1.41 \pm 0.05^{a}$ & $1.28 \pm 0.05^{\mathrm{ab}}$ & $1.33 \pm 0.01^{\mathrm{ab}}$ & 0.03 \\
\hline Isoleucine & $2.19 \pm 0.03^{c}$ & $2.32 \pm 0.01^{b c}$ & $2.58 \pm 0.04^{\mathrm{abc}}$ & $2.54 \pm 0.07 \mathrm{abc}$ & $2.63 \pm 0.13^{a b}$ & $2.46 \pm 0.10^{a b c}$ & $2.78 \pm 0.12^{\mathrm{a}}$ & 0.00 \\
\hline Leucine & $3.89 \pm 0.03^{d}$ & $4.17 \pm 0.03^{\mathrm{cd}}$ & $4.15 \pm 0.05^{\mathrm{cd}}$ & $4.78 \pm 0.18^{\mathrm{ab}}$ & $4.65 \pm 0.01^{\mathrm{ab}}$ & $4.58 \pm 0.12^{b c}$ & $5.10 \pm 0.11^{\mathrm{a}}$ & 0.00 \\
\hline Lysine & $4.52 \pm 0.08^{b}$ & $4.52 \pm 0.02^{b}$ & $4.52 \pm 0.05^{b}$ & $4.82 \pm 0.01^{b}$ & $4.85 \pm 0.08^{b}$ & $5.22 \pm 0.09^{a}$ & $5.43 \pm 0.10^{\mathrm{a}}$ & 0.00 \\
\hline Methionine & $1.50 \pm 0.04^{b}$ & $1.61 \pm 0.04^{\mathrm{ab}}$ & $1.68 \pm 0.07^{\mathrm{ab}}$ & $1.83 \pm 0.06^{\mathrm{a}}$ & $1.63 \pm 0.05^{\mathrm{ab}}$ & $1.78 \pm 0.10^{\mathrm{a}}$ & $1.86 \pm 0.01^{a}$ & 0.01 \\
\hline Phenylalanine & $2.21 \pm 0.09^{b}$ & $2.36 \pm 0.04^{\mathrm{ab}}$ & $2.50 \pm 0.03^{\mathrm{ab}}$ & $2.57 \pm 0.05^{\mathrm{ab}}$ & $2.70 \pm 0.14^{\mathrm{ab}}$ & $2.95 \pm 0.30^{\mathrm{a}}$ & $2.76 \pm 0.03^{a b}$ & 0.02 \\
\hline Threonine & $2.48 \pm 0.09^{b}$ & $2.33 \pm 0.01^{b}$ & $2.30 \pm 0.03^{b}$ & $2.74 \pm 0.05^{a}$ & $2.77 \pm 0.01^{a}$ & $2.86 \pm 0.04^{a}$ & $2.88 \pm 0.02^{\mathrm{a}}$ & 0.00 \\
\hline Valine & $2.39 \pm 0.07^{b}$ & $2.47 \pm 0.01^{\mathrm{b}}$ & $2.84 \pm 0.06^{a}$ & $2.41 \pm 0.07^{b}$ & $2.74 \pm 0.05^{\mathrm{ab}}$ & $2.58 \pm 0.02^{b c}$ & $2.95 \pm 0.02^{\mathrm{a}}$ & 0.00 \\
\hline \multicolumn{9}{|l|}{ NEAA } \\
\hline Alanine & $2.82 \pm 0.03$ & $2.95 \pm 0.04$ & $2.97 \pm 0.01$ & $2.92 \pm 0.08$ & $2.92 \pm 0.04$ & $3.20 \pm 0.27$ & $3.21 \pm 0.03$ & 0.18 \\
\hline Aspartic acid & $4.88 \pm 0.05^{c}$ & $4.98 \pm 0.05^{b c}$ & $4.93 \pm 0.03^{c}$ & $5.30 \pm 0.01^{\mathrm{abc}}$ & $5.12 \pm 0.01^{b c}$ & $5.48 \pm 0.14^{\mathrm{ab}}$ & $5.75 \pm 0.25^{a}$ & 0.00 \\
\hline Cysteine & $0.44 \pm 0.01$ & $0.46 \pm 0.01$ & $0.49 \pm 0.03$ & $0.46 \pm 0.03$ & $0.46 \pm 0.02$ & $0.48 \pm 0.03$ & $0.45 \pm 0.01$ & 0.79 \\
\hline Glutamic acid & $7.47 \pm 0.06^{\mathrm{e}}$ & $7.63 \pm 0.04^{\mathrm{de}}$ & $7.64 \pm 0.08^{\mathrm{de}}$ & $8.13 \pm 0.21^{\mathrm{cd}}$ & $8.91 \pm 0.01^{b}$ & $8.48 \pm 0.06^{b c}$ & $9.63 \pm 0.16^{a}$ & 0.00 \\
\hline Glycine & $2.49 \pm 0.04^{c}$ & $2.75 \pm 0.23^{b c}$ & $3.07 \pm 0.04^{\mathrm{ab}}$ & $3.09 \pm 0.15^{\mathrm{ab}}$ & $3.03 \pm 0.02^{\mathrm{ab}}$ & $2.90 \pm 0.05^{\mathrm{abc}}$ & $3.29 \pm 0.01^{\mathrm{a}}$ & 0.00 \\
\hline Proline & $1.64 \pm 0.03^{b}$ & $1.69 \pm 0.05^{b}$ & $1.85 \pm 0.07^{\mathrm{ab}}$ & $2.15 \pm 0.00^{\mathrm{ab}}$ & $2.18 \pm 0.02^{\mathrm{ab}}$ & $2.34 \pm 0.18^{a}$ & $1.90 \pm 0.22^{\mathrm{ab}}$ & 0.00 \\
\hline Serine & $2.20 \pm 0.05^{\mathrm{ab}}$ & $2.18 \pm 0.01^{\mathrm{ab}}$ & $2.09 \pm 0.02^{b}$ & $2.33 \pm 0.06^{\mathrm{ab}}$ & $2.36 \pm 0.01^{b}$ & $2.39 \pm 0.09^{b}$ & $2.65 \pm 0.05^{a}$ & 0.00 \\
\hline Tyrosine & $1.75 \pm 0.05^{c}$ & $1.81 \pm 0.06^{b c}$ & $1.92 \pm 0.09^{a b c}$ & $2.11 \pm 0.10^{\mathrm{ab}}$ & $1.99 \pm 0.01^{a b c}$ & $2.09 \pm 0.07^{\mathrm{ab}}$ & $2.19 \pm 0.03^{a}$ & 0.00 \\
\hline TAA & $47.36 \pm 0.37^{d}$ & $48.71 \pm 0.33^{d}$ & $50.12 \pm 0.16^{\mathrm{cd}}$ & $52.82 \pm 1.12^{\mathrm{bc}}$ & $53.91 \pm 0.40^{b}$ & $54.54 \pm 1.42^{\mathrm{ab}}$ & $58.21 \pm 0.73^{a}$ & 0.00 \\
\hline EAA & $23.67 \pm 0.28^{d}$ & $24.27 \pm 0.12^{d}$ & $25.15 \pm 0.07^{\mathrm{cd}}$ & $26.32 \pm 0.61^{b c}$ & $26.95 \pm 0.34^{b c}$ & $27.20 \pm 0.67^{a b}$ & $29.13 \pm 0.41^{a}$ & 0.00 \\
\hline NEAA & $23.69 \pm 0.12^{d}$ & $24.45 \pm 0.22^{d}$ & $24.97 \pm 0.10^{c d}$ & $26.50 \pm 0.52^{b c}$ & $26.97 \pm 0.06^{b}$ & $27.34 \pm 0.75^{\mathrm{ab}}$ & $29.08 \pm 0.33^{a}$ & 0.00 \\
\hline EAA/NEAA & $1.00 \pm 0.01$ & $0.99 \pm 0.01$ & $1.01 \pm 0.01$ & $0.99 \pm 0.01$ & $1.00 \pm 0.01$ & $0.99 \pm 0.01$ & $1.00 \pm 0.01$ & 0.71 \\
\hline
\end{tabular}

\section{Skin colour parameters}

As shown in Table 10 and Figure 1, the skin colour of dorsal and caudal regions was not significantly affected by dietary treatments $(P>0.05)$. With increasing dietary TM meal levels, the redness $\left(\mathrm{a}^{*}\right)$ in the ventral regions and bottom of ventral of fish showed an obviously decreasing trend and the lowest value was found in the TM60 group, while the yellowness $\left(\mathrm{b}^{*}\right)$ significantly increased and the maximum value appeared in TM60 group $(P<0.05)$. The lightness $\left(L^{*}\right)$ did not show significant difference among all the treatments $(P>0.05)$.

\section{Muscle $\mathrm{pH}$, water holding capacity and texture}

The $\mathrm{pH}, \mathrm{WHC}$ and texture parameters of muscle are presented in Table 11. No significant difference was observed for $\mathrm{pH}$ and cooking loss among all the groups
$(P>0.05)$. The drip loss significantly increased in TM75 and TM100 groups compared with that in the control group $(P<0.05)$. The muscle hardness, springiness, chewiness and gumminess tend to decrease in TM75 and TM100 groups, however, there were no significant differences $(P>0.05)$. The TM45 group had a higher muscle adhesiveness than that in TM0 group $(P<0.05)$. Shear force in TM75 and TM100 groups were significantly lower than that in the control group $(P<0.05)$.

\section{Muscle histology}

As shown in Figure 2, muscle cellularity was affected by the different levels of TM meal in the diets. The TM100 group showed a higher myofibre diameter and lower myofibre density compared to the control group $(P<0.05)$. More intuitive results are shown with micrographs of muscle tissue sections. 
Table 8. Effects of fishmeal replacement by TM meal on fatty acid composition of large yellow croaker (\% total fatty acids)., ${ }^{1,2}$

Diets

\begin{tabular}{llllllll}
\hline TM0 & TM15 & TM30 & TM45 & TM60 & TM75 & TM100 & $P$-value
\end{tabular}

Fatty acid

\begin{tabular}{|c|c|c|c|c|c|c|c|c|}
\hline $\mathrm{C} 14: 0$ & $4.19 \pm 0.07$ & $4.16 \pm 0.01$ & $4.12 \pm 0.13$ & $4.02 \pm 0.04$ & $3.99 \pm 0.03$ & $4.10 \pm 0.15$ & $4.40 \pm 0.01$ & 0.06 \\
\hline $\mathrm{C} 16: 0$ & $52.48 \pm 0.20$ & $52.87 \pm 0.27$ & $51.34 \pm 0.52$ & $51.62 \pm 0.50$ & $51.38 \pm 0.21$ & $51.62 \pm 0.85$ & $51.93 \pm 0.23$ & 0.22 \\
\hline C18:0 & $12.25 \pm 0.14$ & $12.44 \pm 0.10$ & $12.70 \pm 0.24$ & $12.99 \pm 0.21$ & $12.88 \pm 0.27$ & $12.56 \pm 0.58$ & $12.87 \pm 0.09$ & 0.51 \\
\hline C20:0 & $0.43 \pm 0.04^{b}$ & $0.59 \pm 0.06^{\mathrm{ab}}$ & $0.60 \pm 0.06^{\mathrm{ab}}$ & $0.58 \pm 0.01^{\mathrm{ab}}$ & $0.66 \pm 0.04^{a b}$ & $0.62 \pm 0.06^{\mathrm{ab}}$ & $0.79 \pm 0.07^{a}$ & 0.01 \\
\hline C16:n-7 & $4.73 \pm 0.00^{\mathrm{ab}}$ & $4.76 \pm 0.01^{a}$ & $4.62 \pm 0.05^{\mathrm{abc}}$ & $4.54 \pm 0.02^{\mathrm{bcd}}$ & $4.42 \pm 0.06^{\mathrm{cd}}$ & $4.82 \pm 0.09^{a}$ & $4.33 \pm 0.02^{d}$ & 0.00 \\
\hline C18:n-9 & $4.32 \pm 0.18$ & $4.10 \pm 0.10$ & $4.48 \pm 0.17$ & $4.11 \pm 0.02$ & $3.98 \pm 0.06$ & $4.54 \pm 0.17$ & $4.36 \pm 0.04$ & 0.05 \\
\hline$C 18: 2 n-6$ & $10.28 \pm 0.02^{c}$ & $10.47 \pm 0.18^{c}$ & $11.15 \pm 0.25^{b c}$ & $11.67 \pm 0.24^{a b}$ & $11.65 \pm 0.10^{\mathrm{ab}}$ & $10.81 \pm 0.26^{b c}$ & $12.39 \pm 0.32^{\mathrm{a}}$ & 0.00 \\
\hline C20:n-9 & $1.31 \pm 0.00^{\mathrm{ab}}$ & $1.04 \pm 0.14^{b}$ & $1.06 \pm 0.15^{b}$ & $1.22 \pm 0.12^{b}$ & $1.26 \pm 0.15^{b}$ & $1.37 \pm 0.20^{\mathrm{ab}}$ & $1.90 \pm 0.03^{a}$ & 0.01 \\
\hline$C 18: 3 n-3$ & $1.22 \pm 0.01^{b c}$ & $1.26 \pm 0.02^{\mathrm{abc}}$ & $1.38 \pm 0.03^{a}$ & $1.34 \pm 0.03^{a b}$ & $1.35 \pm 0.03^{\mathrm{ab}}$ & $1.17 \pm 0.05^{c}$ & $0.96 \pm 0.02^{d}$ & 0.00 \\
\hline C20:4n-6 & $0.32 \pm 0.00$ & $0.30 \pm 0.01$ & $0.31 \pm 0.02$ & $0.29 \pm 0.01$ & $0.30 \pm 0.02$ & $0.30 \pm 0.05$ & $0.33 \pm 0.01$ & 0.86 \\
\hline C20:5n-3(EPA) & $2.21 \pm 0.04^{a}$ & $2.00 \pm 0.02^{b}$ & $1.98 \pm 0.03^{b}$ & $1.79 \pm 0.01^{c}$ & $1.72 \pm 0.02^{\mathrm{c}}$ & $1.69 \pm 0.09 c$ & $1.51 \pm 0.01^{d}$ & 0.00 \\
\hline C22:6n-3(DHA) & $3.80 \pm 0.04$ & $3.70 \pm 0.04$ & $3.63 \pm 0.02$ & $3.34 \pm 0.01$ & $3.55 \pm 0.13$ & $3.75 \pm 0.23$ & $3.67 \pm 0.04$ & 0.12 \\
\hline Other $\mathrm{FA}^{3}$ & $2.46 \pm 0.10$ & $2.30 \pm 0.03$ & $2.61 \pm 0.09$ & $2.50 \pm 0.06$ & $2.85 \pm 0.40$ & $2.65 \pm 0.25$ & $3.39 \pm 0.37$ & 0.09 \\
\hline$\sum$ SFA & $70.72 \pm 0.35$ & $71.50 \pm 0.29$ & $70.19 \pm 0.61$ & $70.71 \pm 0.24$ & $70.39 \pm 0.55$ & $70.40 \pm 1.31$ & $69.18 \pm 0.57$ & 0.39 \\
\hline$\sum$ MUFA & $10.92 \pm 0.25$ & $10.33 \pm 0.05$ & $10.85 \pm 0.25$ & $10.4 \pm 0.16$ & $10.46 \pm 0.38$ & $11.27 \pm 0.49$ & $11.42 \pm 0.48$ & 0.19 \\
\hline$\sum$ PUFA & $18.37 \pm 0.14$ & $18.17 \pm 0.25$ & $18.95 \pm 0.39$ & $18.9 \pm 0.31$ & $19.15 \pm 0.19$ & $18.32 \pm 0.81$ & $19.40 \pm 0.19$ & 0.27 \\
\hline$\sum n-3$ PUFA & $7.36 \pm 0.09^{a}$ & $7.03 \pm 0.08^{\mathrm{ab}}$ & $7.05 \pm 0.09^{a b}$ & $6.54 \pm 0.05^{b c}$ & $6.69 \pm 0.14^{\mathrm{abc}}$ & $6.66 \pm 0.37^{a b c}$ & $6.05 \pm 0.09^{c}$ & 0.00 \\
\hline$\sum \mathrm{n}-6$ PUFA & $11.01 \pm 0.05^{d}$ & $11.14 \pm 0.18^{\mathrm{cd}}$ & $11.90 \pm 0.30^{\mathrm{bcd}}$ & $12.36 \pm 0.26^{\mathrm{abc}}$ & $12.47 \pm 0.04^{a b}$ & $11.66 \pm 0.45^{\mathrm{bcd}}$ & $13.34 \pm 0.27^{a}$ & 0.00 \\
\hline$\sum n-3 / \sum n-6$ PUFA & $0.67 \pm 0.01^{a}$ & $0.63 \pm 0.01^{\mathrm{ab}}$ & $0.59 \pm 0.01^{b c}$ & $0.53 \pm 0.01^{d}$ & $0.53 \pm 0.01^{d}$ & $0.57 \pm 0.01^{\mathrm{cd}}$ & $0.45 \pm 0.02^{e}$ & 0.00 \\
\hline
\end{tabular}

${ }^{1}$ DHA = docosahexaenoic acid; EPA = eicosapentaenoic acid; MUFA = mono-unsaturated fatty acids; $n-3=n-3$ fatty acids; $n-6=n-6$ fatty acids; PUFA = polyunsaturated fatty acids; SFA = saturated fatty acids.

${ }^{2}$ Values are mean \pm SE. Mean values within the same row with different superscripts are significantly different $(P<0.05)$.

${ }^{3}$ Other fatty acid: C15:0, C17:0, C17: n-7, C18:3n-6, C21:0, C20:2n-6, C22:0, C20:3n-6, C22: n-9, C20:3n-3, C23:0, C22:2n-6, C24:0 and C24: n-9 were also detected but not reported in the table for low levels. They were utilised to calculate the fatty acid groups.

Table 9. Effects of fishmeal replacement by TM meal on digestive and absorptive enzymes activity in intestine of large yellow croaker. $^{1,2}$

\begin{tabular}{|c|c|c|c|c|c|c|c|c|}
\hline & \multicolumn{7}{|l|}{ Diets } & \multirow[b]{2}{*}{$P$-value } \\
\hline & TMO & TM15 & TM30 & TM45 & TM60 & TM75 & TM100 & \\
\hline \multicolumn{9}{|l|}{ Digestive enzymes } \\
\hline AMS & $0.22 \pm 0.01^{a}$ & $0.21 \pm 0.01^{\mathrm{ab}}$ & $0.21 \pm 0.01^{a b c}$ & $0.20 \pm 0.00^{\mathrm{abc}}$ & $0.18 \pm 0.01^{b c}$ & $0.18 \pm 0.01^{b c}$ & $0.17 \pm 0.01^{c}$ & 0.00 \\
\hline LPS & $2.44 \pm 0.06^{\mathrm{a}}$ & $2.30 \pm 0.04^{a}$ & $2.35 \pm 0.02^{\mathrm{a}}$ & $2.27 \pm 0.16^{a}$ & $1.94 \pm 0.03^{b}$ & $1.72 \pm 0.02^{\mathrm{bc}}$ & $1.57 \pm 0.03^{c}$ & 0.00 \\
\hline Trypsin ${ }^{3}$ & $38.11 \pm 0.37^{a}$ & $37.96 \pm 0.09^{a}$ & $37.20 \pm 0.67^{a}$ & $35.33 \pm 0.23^{a}$ & $30.33 \pm 0.87^{b}$ & $28.76 \pm 1.19^{b c}$ & $26.00 \pm 0.92^{c}$ & 0.00 \\
\hline Chymotrypsin 3 & $1.07 \pm 0.05^{a}$ & $1.02 \pm 0.06^{\mathrm{a}}$ & $1.02 \pm 0.06^{\mathrm{a}}$ & $0.90 \pm 0.01^{\mathrm{ab}}$ & $0.83 \pm 0.02^{b}$ & $0.74 \pm 0.01^{b}$ & $0.73 \pm 0.03^{b}$ & 0.00 \\
\hline \multicolumn{9}{|c|}{ Absorptive enzymes } \\
\hline CK & $0.38 \pm 0.01^{a}$ & $0.35 \pm 0.01^{\mathrm{ab}}$ & $0.35 \pm 0.01^{\mathrm{ab}}$ & $0.31 \pm 0.02^{b}$ & $0.26 \pm 0.01^{c}$ & $0.25 \pm 0.01^{c}$ & $0.24 \pm 0.01^{c}$ & 0.00 \\
\hline V-GT & $4.74 \pm 0.23^{a}$ & $4.61 \pm 0.07^{a b}$ & $4.29 \pm 0.04^{\mathrm{ab}}$ & $3.85 \pm 0.17^{b c}$ & $3.46 \pm 0.15^{c d}$ & $2.87 \pm 0.25^{d}$ & $2.71 \pm 0.22^{d}$ & 0.00 \\
\hline $\mathrm{Na}^{+} \mathrm{K}^{+}-\mathrm{ATPase}^{3}$ & $0.94 \pm 0.09^{a}$ & $0.90 \pm 0.04^{a}$ & $0.88 \pm 0.01^{\mathrm{ab}}$ & $0.82 \pm 0.02^{\mathrm{abc}}$ & $0.74 \pm 0.02^{\mathrm{abc}}$ & $0.67 \pm 0.05^{\mathrm{bc}}$ & $0.63 \pm 0.02^{c}$ & 0.00 \\
\hline AKP & $5.39 \pm 0.12^{\mathrm{a}}$ & $5.26 \pm 0.01^{a}$ & $5.21 \pm 0.03^{a}$ & $5.01 \pm 0.09^{a}$ & $4.50 \pm 0.18^{b}$ & $4.20 \pm 0.04^{b}$ & $4.13 \pm 0.07^{b}$ & 0.00 \\
\hline
\end{tabular}


Table 10. Effects of fishmeal replacement by TM meal on skin colour parameters of large yellow croaker. ${ }^{1,2}$

\begin{tabular}{|c|c|c|c|c|c|c|c|c|}
\hline & \multicolumn{7}{|l|}{ Diets } & \multirow[b]{2}{*}{$P$-value } \\
\hline & TMO & TM15 & TM30 & TM45 & TM60 & TM75 & TM100 & \\
\hline \multicolumn{9}{|c|}{ Dorsal region 1} \\
\hline$L^{*}$ & $46.68 \pm 1.60$ & $50.80 \pm 1.81$ & $47.23 \pm 1.54$ & $49.78 \pm 1.61$ & $47.34 \pm 1.59$ & $46.96 \pm 2.10$ & $49.88 \pm 2.22$ & 0.49 \\
\hline$a^{*}$ & $-0.02 \pm 0.62$ & $0.07 \pm 0.47$ & $-0.70 \pm 0.50$ & $0.40 \pm 0.56$ & $-1.02 \pm 0.45$ & $-1.10 \pm 0.51$ & $-1.49 \pm 0.74$ & 0.13 \\
\hline$b^{*}$ & $10.00 \pm 1.26$ & $9.26 \pm 0.68$ & $10.87 \pm 0.98$ & $9.43 \pm 0.21$ & $10.62 \pm 0.92$ & $11.8 \pm 0.59$ & $11.03 \pm 0.86$ & 0.29 \\
\hline \multicolumn{9}{|c|}{ Dorsal region 2} \\
\hline$L^{*}$ & $63.66 \pm 2.05$ & $63.78 \pm 1.82$ & $61.10 \pm 2.75$ & $64.86 \pm 3.13$ & $66.99 \pm 3.26$ & $69.4 \pm 2.89$ & $70.21 \pm 2.05$ & 0.23 \\
\hline$a^{*}$ & $-0.31 \pm 0.50$ & $-0.12 \pm 0.31$ & $-0.79 \pm 0.66$ & $0.10 \pm 0.40$ & $-0.30 \pm 0.24$ & $-0.84 \pm 0.29$ & $-0.53 \pm 0.39$ & 0.68 \\
\hline$b^{*}$ & $14.94 \pm 0.66$ & $14.87 \pm 0.85$ & $14.48 \pm 0.94$ & $13.75 \pm 0.67$ & $14.23 \pm 1.12$ & $13.55 \pm 0.83$ & $14.84 \pm 0.93$ & 0.85 \\
\hline \multicolumn{9}{|c|}{ Caudal region } \\
\hline$L^{*}$ & $84.51 \pm 1.19$ & $84.65 \pm 1.10$ & $85.53 \pm 0.76$ & $85.59 \pm 1.27$ & $85.30 \pm 1.99$ & $88.50 \pm 0.56$ & $86.32 \pm 0.35$ & 0.16 \\
\hline$a^{*}$ & $-3.35 \pm 0.68$ & $-3.13 \pm 0.55$ & $-3.74 \pm 0.98$ & $-4.17 \pm 0.71$ & $-3.93 \pm 1.16$ & $-3.98 \pm 0.94$ & $-4.18 \pm 0.67$ & 0.95 \\
\hline$b^{*}$ & $28.56 \pm 1.86$ & $23.53 \pm 1.47$ & $26.75 \pm 1.51$ & $23.27 \pm 1.93$ & $29.08 \pm 2.24$ & $25.32 \pm 2.81$ & $24.60 \pm 1.22$ & 0.12 \\
\hline \multicolumn{9}{|c|}{ Ventral region 1} \\
\hline$L^{*}$ & $89.88 \pm 0.72$ & $89.57 \pm 0.79$ & $90.88 \pm 1.10$ & $91.53 \pm 1.22$ & $89.76 \pm 0.83$ & $88.78 \pm 0.83$ & $88.65 \pm 1.49$ & 0.40 \\
\hline$a^{*}$ & $-5.67 \pm 1.18^{a b}$ & $-4.14 \pm 1.01^{a}$ & $-3.46 \pm 0.61^{a}$ & $-4.81 \pm 1.03^{a b}$ & $-8.30 \pm 0.47^{b}$ & $-7.09 \pm 0.87^{a b}$ & $-7.07 \pm 0.39^{\mathrm{ab}}$ & 0.00 \\
\hline$b^{*}$ & $32.75 \pm 4.09^{a b c}$ & $24.53 \pm 2.94^{\mathrm{bc}}$ & $21.86 \pm 1.59^{c}$ & $24.91 \pm 4.38^{b c}$ & $38.25 \pm 2.91^{a}$ & $36.85 \pm 3.45^{\mathrm{ab}}$ & $34.6 \pm 2.08^{a b c}$ & 0.00 \\
\hline \multicolumn{9}{|c|}{ Ventral region 2} \\
\hline$L^{*}$ & $90.04 \pm 0.57$ & $89.31 \pm 0.95$ & $89.61 \pm 0.89$ & $90.73 \pm 0.89$ & $88.75 \pm 1.31$ & $90.72 \pm 0.28$ & $89.77 \pm 0.68$ & 0.52 \\
\hline$a^{*}$ & $-5.33 \pm 0.47^{a}$ & $-4.74 \pm 0.39 a$ & $-5.13 \pm 0.89^{a}$ & $-4.95 \pm 0.55^{a}$ & $-8.22 \pm 0.34^{b}$ & $-5.95 \pm 0.86^{\mathrm{ab}}$ & $-6.11 \pm 0.53^{a b}$ & 0.00 \\
\hline$b^{*}$ & $27.64 \pm 1.14^{\mathrm{ab}}$ & $24.53 \pm 1.82^{\mathrm{ab}}$ & $26.11 \pm 1.71^{\mathrm{ab}}$ & $23.21 \pm 1.63^{b}$ & $34.40 \pm 0.98^{a}$ & $27.14 \pm 4.01^{\mathrm{ab}}$ & $29.29 \pm 3.11^{\mathrm{ab}}$ & 0.04 \\
\hline \multicolumn{9}{|c|}{ Bottom of ventral } \\
\hline $\mathrm{L}^{*}$ & $78.54 \pm 0.63$ & $78.50 \pm 0.78$ & $78.62 \pm 0.98$ & $80.33 \pm 1.08$ & $81.33 \pm 0.62$ & $80.41 \pm 0.90$ & $80.41 \pm 0.58$ & 0.60 \\
\hline$a^{*}$ & $-0.05 \pm 1.21^{a b}$ & $0.35 \pm 1.30^{\mathrm{a}}$ & $0.00 \pm 1.47^{a}$ & $-2.77 \pm 0.86^{a b}$ & $-6.57 \pm 0.84^{b}$ & $-3.34 \pm 1.06^{a b}$ & $-5.76 \pm 0.86^{b}$ & 0.01 \\
\hline$b^{*}$ & $47.11 \pm 5.28^{a b}$ & $37.25 \pm 5.17^{b}$ & $47.52 \pm 5.48^{\mathrm{ab}}$ & $50.52 \pm 3.51^{\mathrm{ab}}$ & $62.69 \pm 5.11^{a}$ & $52.50 \pm 4.40^{\mathrm{ab}}$ & $57.64 \pm 3.39^{a}$ & 0.01 \\
\hline
\end{tabular}

\section{Muscle nucleotides content}

There were no significant differences in the muscle content of ATP, ADP, AMP, Hx and Ino among all the treatments $(P>0.05)$ (Table 12). Fish in the TM75 and TM100 groups had significantly lower IMP content than that in the control group $(P<0.05)$. The $\mathrm{K}$ and $\mathrm{Ki}$ values in TM100 group were significantly higher than those in the other groups $(P<0.05)$.

\section{Muscle free amino acids}

According to Table 13, nineteen kinds of free amino acids were detected in muscle. The content of total free amino acids was significantly decreased with the substitution level increasing $(P<0.05)$. It is known that free amino acids contributed to the formation of five tastes (Kong et al., 2017; Merlo et al., 2021; Zhu et al., 2021). The contents of sweet amino acids (Gly, Ala, Ser, Thr, Pro) and sour amino acids (Glu, Asp, His) significantly decreased when the proportion of substitution over $45 \%(P<0.05)$. The content of umami taste amino acids (Glu, Asp, Gly, Ala, Phe, Tyr) increased first and then decreased, and the lowest value appeared in the TM100 group, while the content of salty taste amino acids (Glu, Asp) showed an opposite trend with the increase of TM meal inclusion $(P<0.05)$. The content of bitter taste amino acids (Met, Val, Ile, Leu, Arg) was not significantly affected by dietary treatments $(P>0.05)$.

\section{Identification of volatile compounds in muscle}

As shown in Figure 3, all information given by the fingerprint analysis technique was used to qualitatively characterise. The relative contents of volatile components were measured by peak volume normalisation (Table 14 ), seventeen volatile compounds were detected by the GC-IMS Library. Among them, 7 aldehydes, 6 ketones, 1 ester, 1 alcohol, 1 acid and 1 phenol were identified. The control group showed higher trans-2-pentenal, 

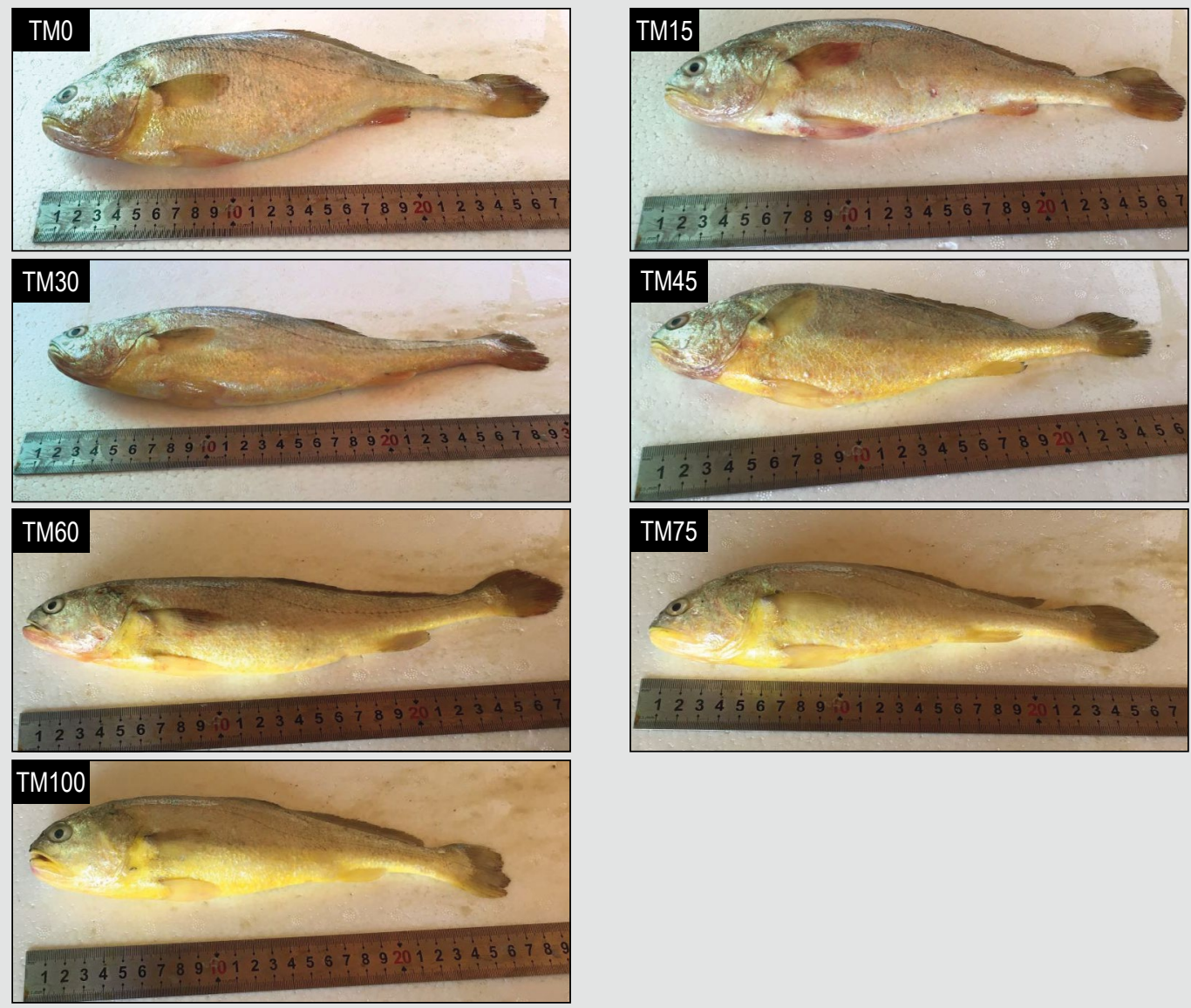

Figure 1. Large yellow croaker fed with different experimental diets after an 80-day feeding trial to show the skin colour.

Table 11. Muscle $\mathrm{pH}$, water holding capacity and texture parameters of large yellow croaker. ${ }^{1}$

\begin{tabular}{|c|c|c|c|c|c|c|c|c|}
\hline & \multicolumn{7}{|l|}{ Diets } & \multirow[b]{2}{*}{$P$-value } \\
\hline & TMO & TM15 & TM30 & TM45 & TM60 & TM75 & TM100 & \\
\hline $\mathrm{pH}$ & $7.02 \pm 0.03$ & $7.08 \pm 0.05$ & $7.02 \pm 0.04$ & $7.07 \pm 0.03$ & $7.05 \pm 0.03$ & $7.07 \pm 0.02$ & $7.04 \pm 0.08$ & 0.93 \\
\hline Cooking loss (\%) & $7.29 \pm 0.81$ & $8.69 \pm 0.62$ & $9.14 \pm 1.03$ & $9.49 \pm 0.87$ & $9.36 \pm 0.54$ & $10.08 \pm 0.89$ & $10.74 \pm 0.93$ & 0.16 \\
\hline Drip loss (\%) & $1.98 \pm 0.05^{b}$ & $2.46 \pm 0.15^{\mathrm{ab}}$ & $2.69 \pm 0.09^{\mathrm{ab}}$ & $2.62 \pm 0.13^{\mathrm{ab}}$ & $2.75 \pm 0.12^{2 \mathrm{~b}}$ & $3.26 \pm 0.25^{a}$ & $3.24 \pm 0.29^{\mathrm{a}}$ & 0.00 \\
\hline \multicolumn{9}{|l|}{ Texture parameters } \\
\hline Hardness (N) & $5.75 \pm 0.52$ & $5.76 \pm 0.27$ & $5.68 \pm 0.33$ & $6.20 \pm 0.39$ & $4.92 \pm 0.22$ & $5.31 \pm 0.31$ & $4.83 \pm 0.49$ & 0.17 \\
\hline Springiness (mm) & $1.43 \pm 0.08$ & $1.32 \pm 0.09$ & $1.41 \pm 0.09$ & $1.45 \pm 0.07$ & $1.32 \pm 0.05$ & $1.29 \pm 0.05$ & $1.11 \pm 0.11$ & 0.07 \\
\hline Chewiness (mJ) & $1.71 \pm 0.22$ & $1.58 \pm 0.09$ & $1.75 \pm 0.15$ & $2.07 \pm 0.25$ & $1.54 \pm 0.12$ & $1.55 \pm 0.11$ & $1.24 \pm 0.27$ & 0.14 \\
\hline Cohesiveness & $0.21 \pm 0.01$ & $0.21 \pm 0.01$ & $0.22 \pm 0.01$ & $0.22 \pm 0.01$ & $0.24 \pm 0.01$ & $0.23 \pm 0.01$ & $0.21 \pm 0.01$ & 0.39 \\
\hline Adhesiveness ( $\left.\mathrm{N}^{*} \mathrm{~mm}\right)$ & $0.11 \pm 0.01^{b}$ & $0.16 \pm 0.03^{\mathrm{ab}}$ & $0.15 \pm 0.01^{\mathrm{ab}}$ & $0.18 \pm 0.01^{a}$ & $0.17 \pm 0.01^{\mathrm{ab}}$ & $0.17 \pm 0.01^{\mathrm{ab}}$ & $0.16 \pm 0.02^{\mathrm{ab}}$ & 0.03 \\
\hline Gumminess (N) & $1.19 \pm 0.14$ & $1.20 \pm 0.05$ & $1.24 \pm 0.08$ & $1.38 \pm 0.11$ & $1.18 \pm 0.08$ & $1.19 \pm 0.05$ & $1.04 \pm 0.14$ & 0.48 \\
\hline Shear force $(\mathrm{N})$ & $9.14 \pm 0.48^{a}$ & $8.27 \pm 0.54^{\mathrm{ab}}$ & $8.77 \pm 0.20^{\mathrm{ab}}$ & $9.58 \pm 0.93^{a}$ & $8.14 \pm 0.49^{a b}$ & $7.36 \pm 0.23^{b}$ & $7.21 \pm 0.32^{b}$ & 0.02 \\
\hline
\end{tabular}

${ }^{1}$ Values are mean \pm SE. Mean values within the same row with different superscripts are significantly different $(P<0.05)$. 


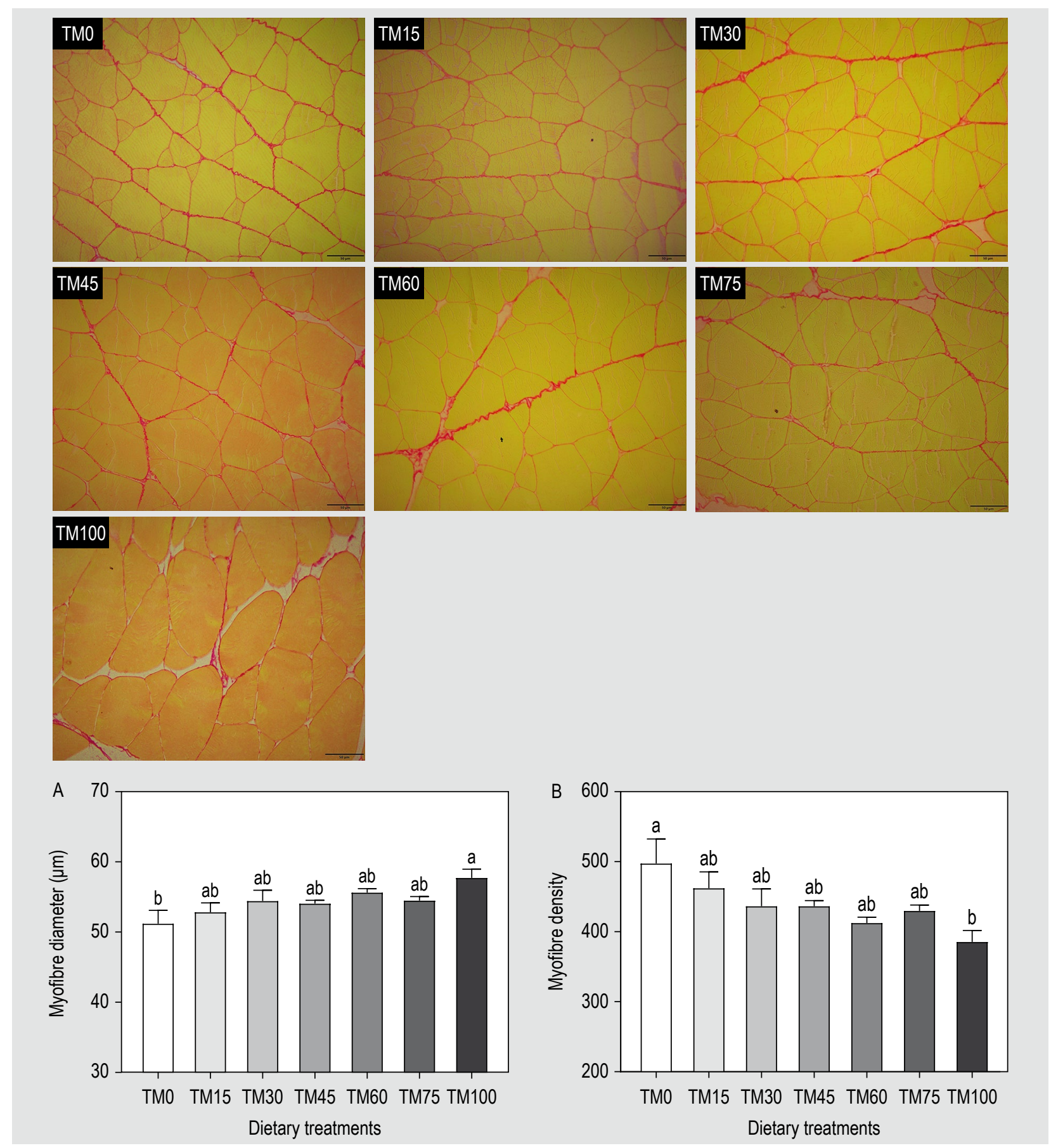

Figure 2. Muscle morphology of large yellow croaker stained with picrosirius red stain (bar=50 $\mu \mathrm{m})$. Muscle cellularity (A): myofibre diameter $(\mu \mathrm{m})$, (B): myofibre density. Data are shown as mean $\pm \mathrm{SE}$. Values with different letters means significant differences $(P<0.05$, Tukey's test).

2-hexanone, hexanal, benzaldehyde, 2-heptanone and pentanal contents compared to other groups. Meanwhile, higher levels of 2-methylbutanal, 3-methylbutanal, acetic acid, methylpropanal and 3-methylbutanol were detected mainly in TM75 and TM100 groups. The relative content of total volatile components increased significantly with the replacement level increasing $(P<0.05)$.

\section{Gene expression in muscle}

The levels of gene expression in muscle are presented in Figure 4. The expression of $M y o D, M y o G, m y f 5$ and Pax-7 decreased generally by the increasing dietary TM meal levels, although no significant differences were detected between groups $(P>0.05)$. The mRNA level of myf6 first increased and then decreased, and the lowest value appeared 
Table 12. Muscle nucleotides content ( $\mu \mathrm{mol} / \mathrm{g}$, wet basis) and freshness (\%) of large yellow croaker. ${ }^{1,2}$

\begin{tabular}{lllllllll}
\multicolumn{7}{c}{ Diets } & \\
\cline { 2 - 6 } & TM0 & TM15 & TM30 & TM45 & TM60 & TM75 & TM100 & $P$-value \\
ATP & $0.09 \pm 0.01$ & $0.09 \pm 0.00$ & $0.08 \pm 0.01$ & $0.12 \pm 0.01$ & $0.11 \pm 0.01$ & $0.11 \pm 0.01$ & $0.10 \pm 0.01$ & 0.09 \\
ADP & $0.18 \pm 0.01$ & $0.17 \pm 0.01$ & $0.18 \pm 0.02$ & $0.19 \pm 0.01$ & $0.19 \pm 0.01$ & $0.19 \pm 0.01$ & $0.18 \pm 0.02$ & 0.62 \\
AMP & $0.42 \pm 0.04$ & $0.41 \pm 0.06$ & $0.49 \pm 0.01$ & $0.33 \pm 0.02$ & $0.36 \pm 0.02$ & $0.38 \pm 0.03$ & $0.37 \pm 0.03$ & 0.09 \\
IMP & $6.06 \pm 0.03^{\mathrm{a}}$ & $5.83 \pm 0.08^{\mathrm{ab}}$ & $5.99 \pm 0.14^{\mathrm{ab}}$ & $5.69 \pm 0.10^{\mathrm{ab}}$ & $5.76 \pm 0.02^{\mathrm{ab}}$ & $5.54 \pm 0.16^{\mathrm{bc}}$ & $5.12 \pm 0.13^{\mathrm{c}}$ & 0.00 \\
Ino & $0.15 \pm 0.01$ & $0.13 \pm 0.01$ & $0.15 \pm 0.01$ & $0.14 \pm 0.01$ & $0.14 \pm 0.01$ & $0.14 \pm 0.01$ & $0.14 \pm 0.01$ & 0.62 \\
Hx & $0.23 \pm 0.01$ & $0.22 \pm 0.01$ & $0.23 \pm 0.01$ & $0.23 \pm 0.01$ & $0.23 \pm 0.01$ & $0.22 \pm 0.01$ & $0.23 \pm 0.01$ & 0.81 \\
K (\%) & $5.36 \pm 0.11^{\mathrm{b}}$ & $5.17 \pm 0.05^{\mathrm{b}}$ & $5.36 \pm 0.11^{\mathrm{b}}$ & $5.48 \pm 0.15^{\mathrm{b}}$ & $5.52 \pm 0.21^{\mathrm{b}}$ & $5.50 \pm 0.22^{\mathrm{b}}$ & $6.02 \pm 0.15^{\mathrm{a}}$ & 0.04 \\
Ki (\%) & $5.93 \pm 0.12^{\mathrm{b}}$ & $5.72 \pm 0.10^{\mathrm{b}}$ & $5.99 \pm 0.11^{\mathrm{b}}$ & $6.07 \pm 0.16^{\mathrm{b}}$ & $6.11 \pm 0.22^{\mathrm{b}}$ & $6.13 \pm 0.25^{\mathrm{b}}$ & $6.74 \pm 0.18^{\mathrm{a}}$ & 0.03 \\
\hline
\end{tabular}

${ }^{1} \mathrm{ADP}=$ adenosine-5' - diphosphate; $\mathrm{AMP}=$ adenosine-5' - monophosphate; $\mathrm{ATP}=$ adenosine-5' - triphosphate; $\mathrm{Hx}=$ hypoxanthine; IMP = inosine-5'-monophosphate; Ino $=$ inosine; $K(\%)=100 \times[(\operatorname{Ino}+H x) /(A T P+A D P+A M P+I M P+I n o+H x)] ; K i(\%)=100 \times[(I n o+H x) /(I M P+I n o+H x)]$.

2 Values are mean \pm SE. Mean values within the same row with different superscripts are significantly different $(P<0.05)$.

Table 13. Effects of fishmeal replacement by TM meal on muscle free amino acids content of large yellow croaker $(\mathrm{mg} / 100 \mathrm{~g}$, wet basis). ${ }^{1}$

\begin{tabular}{|c|c|c|c|c|c|c|c|c|}
\hline & \multicolumn{7}{|l|}{ Diets } & \multirow[b]{2}{*}{$P$-value } \\
\hline & TMO & TM15 & TM30 & TM45 & TM60 & TM75 & TM100 & \\
\hline Alanine & $11.86 \pm 0.86^{\mathrm{ab}}$ & $12.27 \pm 0.41^{\mathrm{ab}}$ & $10.65 \pm 0.19^{b c}$ & $9.69 \pm 0.77^{b c}$ & $8.53 \pm 0.74^{c}$ & $12.11 \pm 0.69^{\mathrm{ab}}$ & $14.25 \pm 0.66^{a}$ & 0.00 \\
\hline Arginine & $5.50 \pm 0.28^{a}$ & $3.79 \pm 0.33^{\mathrm{ab}}$ & $3.89 \pm 0.27^{a b}$ & $4.10 \pm 0.65^{\mathrm{ab}}$ & $2.87 \pm 0.58^{b}$ & $2.46 \pm 0.12^{\mathrm{bc}}$ & $0.93 \pm 0.18^{c}$ & 0.00 \\
\hline Aspartic acid & $1.02 \pm 0.07^{b c}$ & $1.31 \pm 0.13^{a b c}$ & $0.90 \pm 0.18^{c}$ & $0.90 \pm 0.10^{c}$ & $0.83 \pm 0.04^{c}$ & $1.48 \pm 0.31^{\mathrm{ab}}$ & $1.54 \pm 0.09^{a}$ & 0.02 \\
\hline Cysteine & $6.46 \pm 0.74$ & $6.32 \pm 0.19$ & $5.92 \pm 0.33$ & $6.55 \pm 0.11$ & $6.07 \pm 0.26$ & $6.58 \pm 0.40$ & $6.65 \pm 0.32$ & 0.78 \\
\hline Glutamic acid & $7.14 \pm 0.35^{b}$ & $7.20 \pm 0.40^{b}$ & $5.54 \pm 0.44^{b}$ & $6.97 \pm 0.51^{b}$ & $6.13 \pm 0.24^{b}$ & $7.54 \pm 0.38^{b}$ & $10.15 \pm 0.64^{a}$ & 0.00 \\
\hline Glycine & $100.79 \pm 9.01^{a b c}$ & $131.45 \pm 10.05^{\mathrm{ab}}$ & $140.85 \pm 3.46^{a}$ & $112.69 \pm 11.22^{\mathrm{ab}}$ & $122.70 \pm 11.22^{\mathrm{ab}}$ & $96.05 \pm 8.00^{b c}$ & $58.16 \pm 6.26^{c}$ & 0.00 \\
\hline Histidine & $22.93 \pm 1.57^{a}$ & $21.14 \pm 0.59^{a b}$ & $21.90 \pm 1.29^{a}$ & $19.95 \pm 0.21^{\mathrm{ab}}$ & $16.30 \pm 0.75^{b c}$ & $13.02 \pm 1.43^{c}$ & $6.32 \pm 1.24^{d}$ & 0.00 \\
\hline Hydroxyproline & $15.68 \pm 0.90^{\mathrm{a}}$ & $13.74 \pm 0.63^{\mathrm{ab}}$ & $16.56 \pm 0.76^{a}$ & $10.15 \pm 1.43^{b c}$ & $9.40 \pm 1.68^{b c}$ & $5.58 \pm 0.57^{c d}$ & $2.31 \pm 0.06^{d}$ & 0.00 \\
\hline Isoleucine & $2.14 \pm 0.12^{\mathrm{ab}}$ & $2.26 \pm 0.20^{\mathrm{a}}$ & $1.63 \pm 0.29^{\mathrm{ab}}$ & $1.69 \pm 0.05^{\mathrm{ab}}$ & $1.31 \pm 0.11^{b}$ & $1.87 \pm 0.20^{\mathrm{ab}}$ & $2.25 \pm 0.22^{\mathrm{a}}$ & 0.02 \\
\hline Leucine & $0.77 \pm 0.21$ & $1.38 \pm 0.14$ & $0.95 \pm 0.30$ & $0.71 \pm 0.04$ & $0.87 \pm 0.32$ & $1.31 \pm 0.46$ & $1.77 \pm 0.65$ & 0.36 \\
\hline Lysine & $30.22 \pm 1.42^{\mathrm{a}}$ & $25.87 \pm 0.48^{a b}$ & $22.46 \pm 0.52^{b}$ & $21.31 \pm 1.22^{\mathrm{bc}}$ & $15.93 \pm 1.55^{d}$ & $16.28 \pm 1.31^{\mathrm{cd}}$ & $8.76 \pm 0.58^{e}$ & 0.00 \\
\hline Methionine & $2.31 \pm 0.89$ & $2.51 \pm 0.45$ & $2.87 \pm 0.34$ & $3.38 \pm 0.06$ & $2.95 \pm 0.57$ & $2.07 \pm 0.63$ & $2.61 \pm 0.35$ & 0.67 \\
\hline Phenylalanine & $1.61 \pm 0.39$ & $1.99 \pm 0.32$ & $1.19 \pm 0.09$ & $1.05 \pm 0.09$ & $1.08 \pm 0.04$ & $2.32 \pm 1.38$ & $1.26 \pm 0.17$ & 0.61 \\
\hline Proline & $98.49 \pm 8.57^{a}$ & $60.58 \pm 4.33^{b c}$ & $59.08 \pm 8.96^{b c}$ & $76.80 \pm 2.49^{a b}$ & $45.50 \pm 5.39 \mathrm{~cd}$ & $60.21 \pm 5.28^{b c}$ & $29.50 \pm 3.40^{d}$ & 0.00 \\
\hline Serine & $44.02 \pm 3.93$ & $55.56 \pm 8.02$ & $42.23 \pm 7.58$ & $49.46 \pm 4.21$ & $49.80 \pm 3.25$ & $59.69 \pm 0.95$ & $45.67 \pm 2.46$ & 0.22 \\
\hline Taurine & $89.00 \pm 4.25^{\mathrm{ab}}$ & $71.00 \pm 0.98^{a b}$ & $76.60 \pm 4.29^{a b}$ & $67.71 \pm 4.52^{b}$ & $73.48 \pm 9.72^{\mathrm{ab}}$ & $75.04 \pm 2.68^{a b}$ & $91.90 \pm 3.78^{a}$ & 0.03 \\
\hline Threonine & $49.67 \pm 5.02^{\mathrm{a}}$ & $19.24 \pm 5.46^{\mathrm{ab}}$ & $21.91 \pm 0.86^{b}$ & $15.01 \pm 2.94^{\mathrm{ab}}$ & $11.17 \pm 2.77^{a b}$ & $8.53 \pm 1.74^{\mathrm{ab}}$ & $3.82 \pm 0.28^{c}$ & 0.00 \\
\hline Tyrosine & $3.02 \pm 0.24$ & $3.29 \pm 0.30$ & $2.99 \pm 0.17$ & $2.74 \pm 0.04$ & $3.13 \pm 0.23$ & $2.69 \pm 0.05$ & $2.77 \pm 0.14$ & 0.30 \\
\hline Valine & $5.46 \pm 0.31$ & $5.96 \pm 0.69$ & $5.40 \pm 1.08$ & $6.16 \pm 0.61$ & $4.52 \pm 0.57$ & $4.60 \pm 0.58$ & $4.36 \pm 0.56$ & 0.37 \\
\hline Total FAA & $498.10 \pm 8.38^{a}$ & $446.88 \pm 10.57^{b}$ & $443.52 \pm 17.31^{b}$ & $417.04 \pm 18.37^{b c}$ & $382.55 \pm 14.68^{c}$ & $379.44 \pm 3.99^{c}$ & $294.96 \pm 12.49^{d}$ & 0.00 \\
\hline EAA & $120.61 \pm 3.38^{a}$ & $84.15 \pm 5.62^{b}$ & $82.20 \pm 0.69^{b}$ & $73.36 \pm 5.23^{b c}$ & $56.99 \pm 5.10^{\mathrm{cd}}$ & $52.47 \pm 4.93^{d}$ & $32.07 \pm 1.14^{\mathrm{e}}$ & 0.00 \\
\hline NEAA & $272.81 \pm 9.81^{a}$ & $277.99 \pm 14.34^{a}$ & $268.16 \pm 16.43^{a}$ & $265.81 \pm 16.56^{\mathrm{a}}$ & $242.68 \pm 6.96^{a}$ & $246.35 \pm 6.22^{\mathrm{a}}$ & $168.68 \pm 10.87^{b}$ & 0.00 \\
\hline Umami taste $^{2}$ & $125.44 \pm 10.25^{\mathrm{ab}}$ & $157.51 \pm 10.37^{a}$ & $162.12 \pm 3.46^{a}$ & $134.06 \pm 10.12^{\mathrm{a}}$ & $142.40 \pm 10.58^{a}$ & $122.19 \pm 5.81^{\mathrm{ab}}$ & $88.11 \pm 5.23^{b}$ & 0.00 \\
\hline Sweet taste ${ }^{3}$ & $304.83 \pm 11.12^{\mathrm{a}}$ & $279.10 \pm 11.48^{a b}$ & $274.72 \pm 16.23^{\mathrm{ab}}$ & $263.66 \pm 19.91^{\mathrm{ab}}$ & $237.70 \pm 8.68^{b}$ & $236.60 \pm 5.24^{b}$ & $151.40 \pm 11.65^{c}$ & 0.00 \\
\hline Salty taste 4 & $8.17 \pm 0.33^{b c}$ & $8.51 \pm 0.27^{b c}$ & $6.44 \pm 0.56^{c}$ & $7.88 \pm 0.42^{\mathrm{bc}}$ & $6.96 \pm 0.26^{b c}$ & $9.01 \pm 0.40^{b}$ & $11.69 \pm 0.73^{a}$ & 0.00 \\
\hline Bitter taste ${ }^{5}$ & $16.18 \pm 1.48$ & $15.90 \pm 0.46$ & $14.74 \pm 0.91$ & $16.04 \pm 1.31$ & $12.51 \pm 1.87$ & $12.31 \pm 1.58$ & $11.91 \pm 1.41$ & 0.13 \\
\hline Sour taste ${ }^{6}$ & $31.10 \pm 1.25^{a}$ & $29.65 \pm 0.68^{a}$ & $28.34 \pm 0.89^{a}$ & $27.82 \pm 0.26^{\mathrm{ab}}$ & $23.25 \pm 0.99^{b c}$ & $22.03 \pm 1.64^{\mathrm{cd}}$ & $18.01 \pm 0.51^{d}$ & 0.00 \\
\hline
\end{tabular}

\footnotetext{
${ }^{1}$ EAA = essential amino acids; NEAA = non-essential amino acids; Total FAA = total free amino acid content. $\quad 2$ Umami taste: Glu + Asp + Gly + Ala +Phe +Tyr. ${ }^{3}$ Sweet taste: Gly + Ala + Ser + Thr + Pro. ${ }^{4}$ Salty taste: Glu + Asp. ${ }^{5}$ Bitter taste: Met + Val + lle + Leu + Arg. ${ }^{6}$ Sour taste: Glu + Asp + His.
} 


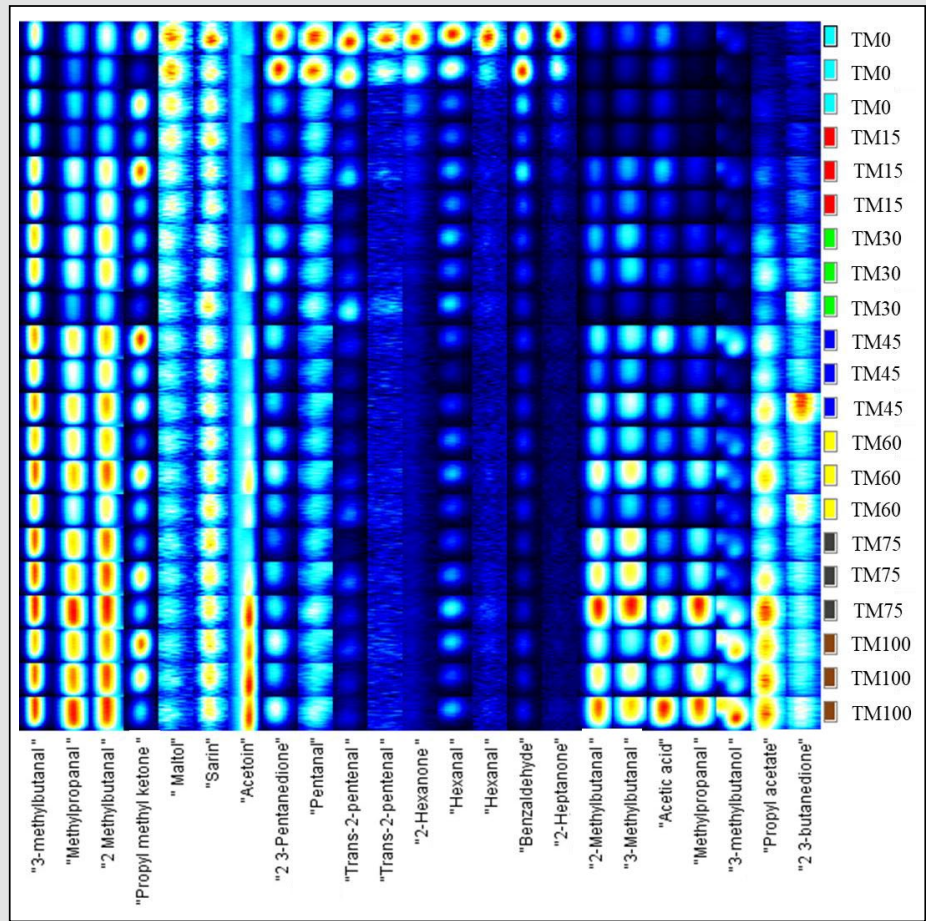

Figure 3. Fingerprints of all the samples generated by Gallery plot. Each row represents a sample $(n=3)$, and each column represents a signal peak for a compound. Some compounds show two peaks, corresponding to monomers and dimers. The colours of the points qualitatively represent the concentration of the substance: light blue indicates low concentration, and red indicates greater concentration.

in the TM100 group $(P<0.05)$. The transcription level of mstn in the TM75 and TM100 groups were significantly higher than those in the groups of TM15, TM30, TM45 $(P>0.05)$.

\section{Discussion}

The TM meal as substitute for fishmeal has been studied in a variety of aquaculture animal species. However, there are no reports on the effects of dietary TM meal on growth performance of large yellow croaker. The present study found that replacement of fishmeal by TM meal had no significant effect on SR, and the TM meal could replace at least $30 \%$ of dietary fishmeal protein without significantly negative effect on the growth of large yellow croaker. Similarly, the African catfish showed equal growth performance when TM meal was used to replace $40 \%$ of dietary fishmeal compared with control group ( $\mathrm{Ng}$ et al., 2001). And previous study in European sea bass juveniles found that full-fat TM meal could be used $25 \%$ of inclusion in diet without affecting growth performance, while 50\% content led to a worsening fish performance (Gasco et al., 2016). In the present study, the FI decreased in the TM75 and TM100 groups, suggesting that high proportion substitution may affect palatability and thus reduce growth performance. The FCR significantly increased when the replacement level over $45 \%$, indicating that feed utilisation was affected, which may be another reason for reduced growth performance.

The utilisation of nutrients depends on the activities of digestive and absorptive enzymes, which play key roles in breaking down and assimilating food. The exocrine pancreas of fish could synthesis and secrete many digestive enzymes into the intestinal lumen, such AMS, LPS, trypsin and chymotrypsin. Intestinal AKP is considered to be involved in absorption of nutrients, such as lipid, glucose and inorganic phosphate (Villanueva et al., 1997), and $\gamma$-GT is involved in peptide transport (Griffith and Meister, 1980). The $\mathrm{Na}^{+} \mathrm{K}^{+}$-ATPase and CK play an important role in the energy metabolism of cells involved in the transport of phosphate, amino acids or glucose into the cells (Decking et al., 2001; Geering, 1990). The present study showed that the activities of AMS, LPS, trypsin and chymotrypsin significantly decreased when the replacement of dietary fishmeal by TM meal over $45 \%$. At the same time, the activities of AKP, $\gamma-\mathrm{GT}, \mathrm{Na}^{+} \mathrm{K}^{+}$-ATPase and CK decreased following the inclusion of TM meal increasing. These data were consistent with the present results of growth performance, which indicated the high level of TM meal as substitution for fishmeal could reduce the activities of digestive and absorptive enzymes thus impair feed utilisation and growth performance of fishes. Some other research also found that high content of TM meal in 
Table 14. Relative content of muscle volatile compounds of large yellow croaker by GC-IMS. ${ }^{1}$

\begin{tabular}{|c|c|c|c|c|c|c|c|c|}
\hline & \multicolumn{7}{|l|}{ Diets } & \multirow[b]{2}{*}{$P$-value } \\
\hline & TMO & TM15 & TM30 & TM45 & TM60 & TM75 & TM100 & \\
\hline \multicolumn{9}{|l|}{ Aldehydes } \\
\hline 3-methylbutanal & $1.00 \pm 0.06^{c}$ & $1.29 \pm 0.01^{b c}$ & $1.48 \pm 0.01^{b}$ & $1.56 \pm 0.07^{b}$ & $1.61 \pm 0.10^{b}$ & $2.04 \pm 0.04^{a}$ & $1.94 \pm 0.11^{\mathrm{a}}$ & 0.00 \\
\hline Methylpropanal & $1.00 \pm 0.06^{e}$ & $1.28 \pm 0.07^{\mathrm{de}}$ & $1.45 \pm 0.01^{\text {cde }}$ & $1.86 \pm 0.14^{\mathrm{bcd}}$ & $1.94 \pm 0.24^{b c}$ & $2.45 \pm 0.15^{\mathrm{ab}}$ & $2.61 \pm 0.14^{a}$ & 0.00 \\
\hline 2-methylbutanal & $1.00 \pm 0.06^{d}$ & $1.18 \pm 0.10^{\mathrm{cd}}$ & $1.36 \pm 0.12^{\mathrm{bcd}}$ & $1.75 \pm 0.10^{b c}$ & $1.91 \pm 0.10^{\mathrm{ab}}$ & $2.45 \pm 0.20^{\mathrm{a}}$ & $2.35 \pm 0.09^{a}$ & 0.00 \\
\hline Hexanal & $1.00 \pm 0.03^{a}$ & $0.48 \pm 0.04^{b}$ & $0.55 \pm 0.04^{b}$ & $0.35 \pm 0.04^{b}$ & $0.47 \pm 0.05^{b}$ & $0.51 \pm 0.08^{b}$ & $0.50 \pm 0.05^{b}$ & 0.00 \\
\hline Trans-2-pentenal & $1.00 \pm 0.08^{a}$ & $0.47 \pm 0.03^{b}$ & $0.29 \pm 0.01^{b c}$ & $0.27 \pm 0.02^{c}$ & $0.30 \pm 0.04^{b c}$ & $0.29 \pm 0.04^{b c}$ & $0.29 \pm 0.03^{b c}$ & 0.00 \\
\hline Pentanal & $1.00 \pm 0.01^{a}$ & $0.61 \pm 0.01^{b}$ & $0.66 \pm 0.02^{b}$ & $0.51 \pm 0.02^{c}$ & $0.57 \pm 0.02^{b c}$ & $0.59 \pm 0.03^{b c}$ & $0.60 \pm 0.02^{b c}$ & 0.00 \\
\hline Benzaldehyde & $1.00 \pm 0.10^{\mathrm{a}}$ & $0.41 \pm 0.06^{b}$ & $0.30 \pm 0.02^{b}$ & $0.30 \pm 0.01^{b}$ & $0.30 \pm 0.02^{b}$ & $0.25 \pm 0.01^{b}$ & $0.29 \pm 0.01^{b}$ & 0.00 \\
\hline \multicolumn{9}{|l|}{ Ketones } \\
\hline Propyl methyl ketone & $1.00 \pm 0.01$ & $1.00 \pm 0.05$ & $0.68 \pm 0.04$ & $0.82 \pm 0.03$ & $0.87 \pm 0.14$ & $0.86 \pm 0.14$ & $0.81 \pm 0.07$ & 0.17 \\
\hline Acetoin & $1.00 \pm 0.02^{\mathrm{bc}}$ & $0.90 \pm 0.05^{c}$ & $1.06 \pm 0.07^{b c}$ & $1.19 \pm 0.05^{\mathrm{bc}}$ & $1.26 \pm 0.07^{\mathrm{abc}}$ & $1.34 \pm 0.15^{\mathrm{ab}}$ & $1.60 \pm 0.03^{a}$ & 0.00 \\
\hline 2,3-pentanedione & $1.00 \pm 0.01^{a}$ & $0.57 \pm 0.03^{b}$ & $0.57 \pm 0.02^{b}$ & $0.43 \pm 0.04^{b}$ & $0.51 \pm 0.06^{b}$ & $0.47 \pm 0.01^{b}$ & $0.57 \pm 0.07^{b}$ & 0.00 \\
\hline 2-hexanone & $1.00 \pm 0.18^{a}$ & $0.47 \pm 0.04^{b}$ & $0.38 \pm 0.02^{b}$ & $0.35 \pm 0.02^{b}$ & $0.33 \pm 0.01^{b}$ & $0.37 \pm 0.01^{b}$ & $0.34 \pm 0.01^{b}$ & 0.00 \\
\hline 2-heptanone & $1.00 \pm 0.14^{a}$ & $0.42 \pm 0.03^{b}$ & $0.28 \pm 0.02^{b}$ & $0.24 \pm 0.02^{b}$ & $0.26 \pm 0.02^{b}$ & $0.24 \pm 0.01^{b}$ & $0.26 \pm 0.01^{b}$ & 0.00 \\
\hline 2,3-butanedione & $1.00 \pm 0.02^{c}$ & $1.31 \pm 0.03^{b c}$ & $1.86 \pm 0.23^{\mathrm{abc}}$ & $2.09 \pm 0.21^{\mathrm{ab}}$ & $2.19 \pm 0.33^{a}$ & $1.93 \pm 0.07^{\mathrm{ab}}$ & $1.78 \pm 0.14^{\mathrm{abc}}$ & 0.00 \\
\hline \multicolumn{9}{|l|}{ Alcohol } \\
\hline 3-methylbutanol & $1.00 \pm 0.05^{c}$ & $0.97 \pm 0.07^{c}$ & $0.92 \pm 0.04^{c}$ & $2.01 \pm 0.20^{b}$ & $1.92 \pm 0.10^{b}$ & $2.30 \pm 0.17^{b}$ & $3.11 \pm 0.28^{a}$ & 0.00 \\
\hline \multicolumn{9}{|l|}{ Acid } \\
\hline Acetic acid & $1.00 \pm 0.08^{d}$ & $1.08 \pm 0.11^{\mathrm{cd}}$ & $1.14 \pm 0.05^{\mathrm{cd}}$ & $2.25 \pm 0.27^{\mathrm{bc}}$ & $1.98 \pm 0.25^{\mathrm{bcd}}$ & $2.35 \pm 0.39^{b}$ & $4.23 \pm 0.33^{a}$ & 0.00 \\
\hline \multicolumn{9}{|l|}{ Phenol } \\
\hline Maltol & $1.00 \pm 0.04^{a}$ & $0.81 \pm 0.03^{b}$ & $0.71 \pm 0.02^{b c}$ & $0.70 \pm 0.02^{b c}$ & $0.65 \pm 0.04^{c}$ & $0.64 \pm 0.02^{c}$ & $0.68 \pm 0.04^{b c}$ & 0.00 \\
\hline \multicolumn{9}{|l|}{ Ester } \\
\hline Propyl acetate & $1.00 \pm 0.09^{d}$ & $1.52 \pm 0.09^{c d}$ & $2.24 \pm 0.16^{c}$ & $3.52 \pm 0.38^{b}$ & $4.10 \pm 0.15^{\mathrm{ab}}$ & $4.43 \pm 0.40^{\mathrm{ab}}$ & $4.92 \pm 0.17^{a}$ & 0.00 \\
\hline Total & $1.00 \pm 0.04^{\mathrm{cd}}$ & $0.97 \pm 0.04^{d}$ & $1.03 \pm 0.02^{\mathrm{cd}}$ & $1.22 \pm 0.06^{\mathrm{cd}}$ & $1.29 \pm 0.09 b c$ & $1.54 \pm 0.09^{\mathrm{ab}}$ & $1.62 \pm 0.06^{a}$ & 0.00 \\
\hline
\end{tabular}

${ }^{1}$ The relative contents of volatile components were measured by peak volume normalisation (the peak volume of the control group was regarded as 1 ). Values are mean \pm SE. Mean values within the same row with different superscripts are significantly different $(P<0.05)$.

diet could lead to worse feed utilisation. The reason could be the presence of chitin in TM meal, the main component of the insect exoskeleton, which could affect digestibility of the nutrients and feed utilisation (Dias et al., 1998; Henry et al., 2015; Kroeckel et al., 2012; Longvah et al., 2011).

Body condition index is often used to evaluate the health, growth and feeding of fish (Hartman and Margraf, 2006). It is also an important evaluation standard of fish quality. Meanwhile, body condition indices can reflect energy or nutrient reserves, as many studies have shown that they were significantly related to fish proximate composition (Brown and Murphy, 2004; Kaufman et al., 2007; Pangle and Sutton, 2010). Lipid is the main energy storage material in fish (Tocher, 2003). In the present study, CF and hepatosomatic index were positively related to muscle lipid content, which is similar to the results from previous study in walleyes (Kaufman et al., 2007). According to Kroeckel et al. (2012), the decrease of crude lipid content was mainly due to the reduction of feed intake with the increase of replacement level of fish meal with black soldier fly meal, which may also have affected physical indicators. In addition, the content of crude protein in TM100 group was significantly higher than that in the control group in the present study. Similar results were reported in rainbow trout (Belforti et al., 2016), yellow catfish (Su et al., 2017) and giant freshwater prawn (Macrobrachium rosenbergii) (Feng et al., 2019). It is reported that the conversion of dietary protein to muscle is a heavy process that requires lipids as an energy source, resulting in a decrease in lipid content and an increase in protein content in muscle (Xu et al., 2015). This is also a possible explanation and the exact metabolic mechanism needs to be further explored.

Amino acids are precursors of protein synthesis. In the present study, both protein-bound EAA and NEAA in muscle were significantly increased with higher TM meal, which was contrary to the trend of EAA and consistent with the trend of NEAA in feed. However, with the increase of replacement level, both free EAA and NEAA in muscle 

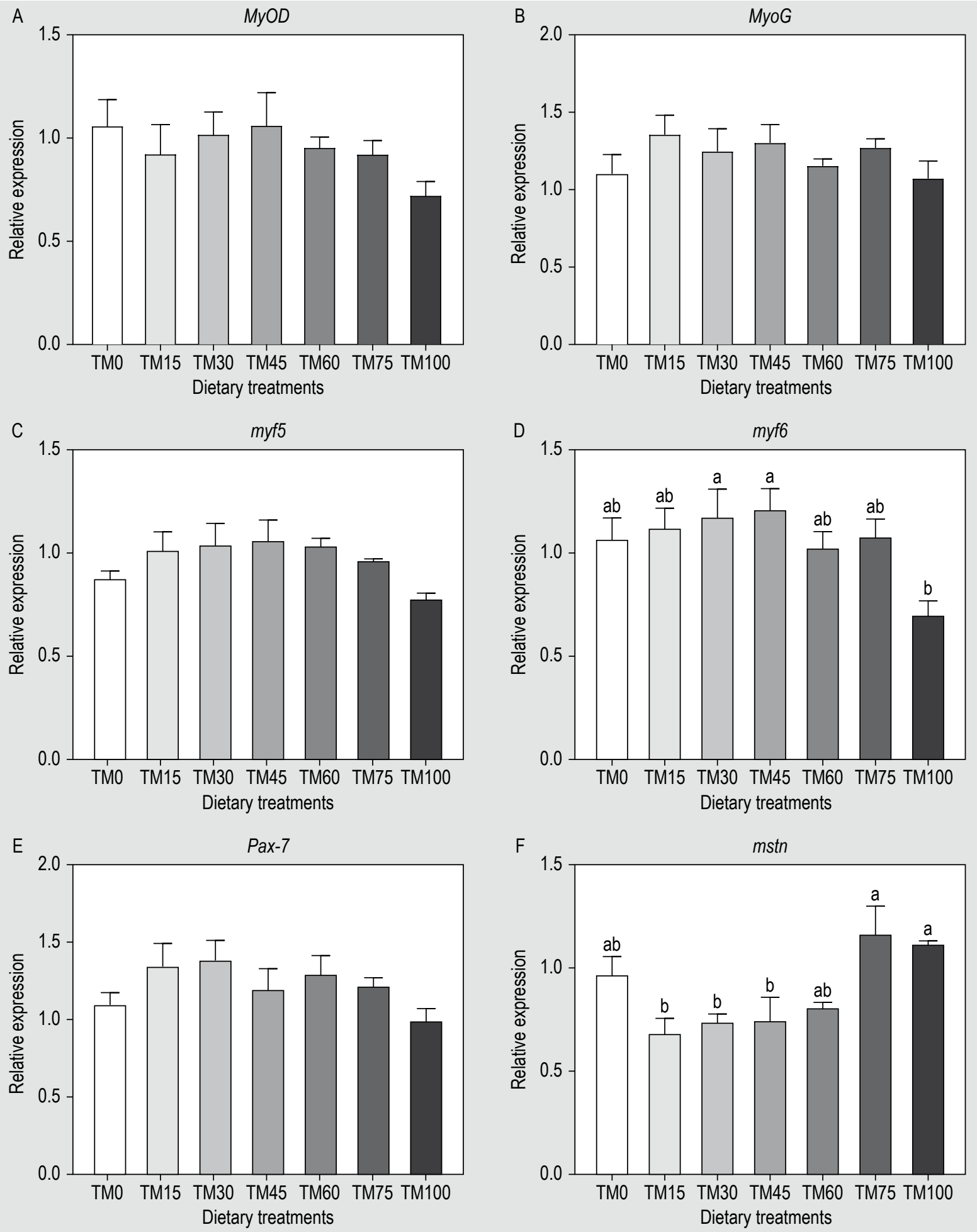

Figure 4. Expression of genes related to the muscle cell growth and differentiation: myogenic regulatory factors (MyoD, MyoG, myf5, myf6) (A-D), Pax-7 (E) and $m s t n(F)$. Data are shown as mean \pm SE. Values with different letters means significant differences $(P<0.05$, Tukey's test).

showed decreasing trend, which were completely contrary to the trend of protein-bound ones. The concentration of free amino acids (FAA) in tissues are mainly controlled by dietary amino acid absorption rate, amino acid oxidation and protein conversion (Carter et al., 1995). According to Carter et al. (1995) and Houlihan et al. (1993), protein synthesis is the main factor in the removal of amino acid from FAA pools, which may explain the decrease trend 
of FAA with the increase of protein-bound amino acids in muscle in the present study. There was no significant difference in crude protein content in muscle except for TM100, which further indicated that protein-bound and free amino acids could complement each other, so that the total amino acid content in muscle did not change significantly. In the research of Iaconisi et al. (2019), replacement of fish meal by TM meal also increased the contents of some essential and non-essential amino acids in muscle of gilthead sea bream and rainbow trout, but it did not provide a clear explanation, indicating that the relationship between amino acid utilisation and digestion and absorption needs further study.

Skin colour and appearance are important factors affecting the market acceptability of aquatic products (Haard, 1992a). Golden yellow skin colour is usually the first direct standard for consumers to evaluate the quality of large yellow croaker. The results in present study showed that the redness $\left(\mathrm{a}^{*}\right)$ gradually decreased while the yellowness $\left(b^{*}\right)$ gradually increased in the ventral regions and the bottom of ventral of fish skin with the dietary TM meal level increasing. It is reported that mealworms contain a variety of carotenoids and riboflavin (Finke, 2002, 2015; Schabel et al., 2010). This may explain that the dietary inclusion of TM meal positively affected skin colour of large yellow croaker, because several studies have shown that the addition of carotenoids to the diet can improve the skin colour of large yellow croaker, such as zeaxanthin, astaxanthin, xanthophylls (Yi et al., 2014), lutein and canthaxanthin (Yi et al., 2016). However, the present results were different from the other study in blackspot sea bream, in which a* value of skin significantly increased while $L^{*}$ value significantly decreased when TM meal replaced 0-50\% fish meal (Iaconisi et al., 2017). It was reported that the effectiveness of carotenoid source in terms of deposition and pigmentation is species specific and skin lightness $\left(L^{*}\right)$ seems to be influenced by environmental factors (Pavlidis et al., 2006), which could partly explain the differences of pigmentation in different fish species.

Fish muscle is the main edible portion (Periago et al., 2005), muscle quality is another factor affecting the market acceptability of fishes which could be affected by nutrient composition. In general, firm, elastic and juicy fish flesh is more acceptable to consumers. Fillet texture is one of the most important criterions to evaluate the muscle quality, of which the shear force is considered to reflect the flesh firmness of fish (Johnston et al., 2006). In the present study, the fillet springiness and shear force tend to decrease with increasing dietary TM meal levels, which indicated that the muscle firmness tended to decrease. It has been reported that the muscle firmness may be positively correlated with collagen and hydroxyproline content (Johnston et al., 2006; Ma et al., 2019; Sato et al., 1986; Wang et al., 2015). In the present study, it was showed that muscle hydroxyproline and collagen content significantly decreased in TM75 and TM100 group. Water-holding capacity is also an important flesh quality parameter reflecting the juicy of fish flesh, which could be evaluated by cooking loss and drip loss. In the present study, it was found that drip loss significantly increased in TM75 and TM100 groups reflecting the poor WHC. Loje et al. (2007) showed that WHC was also positively correlated with collagen and hydroxyproline content. In the present study, the shear force, WHC, hydroxyproline and collagen contents showed the similar downward trend. In addition, $\mathrm{pH}$ is also an important factor affecting flesh quality (Haard, 1992b), the post-mortem $\mathrm{pH}$ decline causes the degradation of connective tissue and softer flesh, however, no dramatic changes were observed in the present study.

Fish muscle is mainly composed of white muscle and red muscle. Muscle growth mainly depends on the hypertrophy and hyperplasia of muscle fibres (Veggetti et al., 1990). The number of muscle fibres is affected by various factors, such as species, strains, diet, and environmental temperature (Ayala et al., 2001; Lopez-Albors et al., 2003), of which diet is an important factor. Muscle cellularity (the number and size distribution of fibres) also affects flesh texture and thus changes the quality of fish (Hurling et al., 1996; Johnston et $a l ., 2000)$. For example, several studies have demonstrated that average muscle fibre diameter is negatively correlated with hardness of flesh (Hatae et al., 1990; Hurling et al., 1996), while the positive correlation between muscle fibre density and textural parameters (such as hardness, springiness) was found by Johnston et al. (2000) and Periago et al. (2005). In the present study, the muscle cellularity was affected by dietary treatments. With increasing replacement levels, muscle fibre diameter gradually increased, while muscle fibre density showed a similar downward trend with texture parameters such as hardness and springiness, which again confirmed above conclusions of previous studies.

Muscle growth is controlled by multiple genes and complex signalling pathways, in which the growth and differentiation of muscle cells are mainly regulated by positive regulatory factors (such as MyoD family) and negative regulatory factors (such as myostatin). In the MyoD family (MyoD, MyoG, myf5 and myf6), MyoD and myf5 are called determinant factor and related to myoblasts proliferation, while $M y o G$ and $m y f 6$ are called differentiation factor and related to myoblasts differentiation and myofiber hypertrophy (Vélez et al., 2017; Weintraub, 1993). Myostatin (mstn), a member of the TGF- $\beta$ superfamily, negatively regulates muscle development by inhibiting the proliferation and differentiation of myogenic progenitor cells (Joulia et al., 2003). Pax7 also plays an important role in muscle development (Mao et al., 2008). Many studies have shown that the expression of $M y o D$ and $M y o G$ are positively correlated with fillet texture (Østbye et al., 2018; Richter et al., 2021). In the present study, total replacement of fishmeal with TM meal down-regulated the expression of $m y f 6$ 
gene, and up-regulated mstn gene expression, indicating that the growth and differentiation of muscle cells may be regulated by feed composition, and mstn gene inhibits the proliferation and differentiation of muscle cells by negatively regulating the transcriptional activity of MyoD family. The results showed that the addition of TM meal could reduce muscle development by regulating genes expression, which resulted in the decrease of muscle fibre density and texture parameters such as firmness and shear force in the high proportion replacement group.

Flavour of fish mainly includes the taste and smell, which is also an important evaluation standard of fish quality for consumers. Volatile components, free amino acids, organic acids, nucleotides and peptides contribute to flavour formation (Konosu, 1979). Taste is made up of five basic qualities: sweetness, saltiness, bitterness, sourness and umami taste (Ninomiya, 1987). According to Fuke and Konosu (1991), free amino acids and nucleotides play important roles in producing the taste. Among them, glutamate and IMP are typical umami taste-active substance (Maruji et al., 2010; Yamaguchi and Ninomiya, 2000). In fish muscle, ATP is degraded through the ATP - ADP - AMP - IMP - Ino - Hx metabolic pathway (OcañoHiguera et al., 2011). In addition, $\mathrm{K}$ and $\mathrm{Ki}$ values were calculated from concentrations of ATP and its degradation products and used to evaluate the freshness of fish, which have a strong correlation with muscle changes in fish after death (Hamada-Sato et al., 2005). The present study showed that the contents of IMP and total free amino acids decreased with the increasing dietary TM meal inclusion level, and $\mathrm{K}$ and Ki values in TM100 group significantly higher than those in the other groups. These results indicated that a higher proportion of substitution had a certain adverse effect on the taste of large yellow croaker. The change of IMP may be related to the decrease of its precursors such as AMP and the activity of related enzymes in the metabolic process. Since there is no significant difference in the content of Ino and $\mathrm{Hx}$, it leads to the decrease of $\mathrm{K}$ and $\mathrm{Ki}$ values. But according to the report of Saito et al. (1959), fillets with K value below $20 \%$ are still very fresh products.

The formation of volatile compounds is mainly through thermal degradation, lipid oxidation and amino acid degradation, the volatile compounds contributing to aromatic odour in fresh fish mainly include volatile carbonyls (aldehydes and ketones) and alcohols, which are obtained from PUFA through lipoxygenase-mediated reactions (Alasalvar et al., 2005). In the present study, aldehydes and ketones were mainly detected in fish fillet. The aromatic smell of fish muscle is derived mainly from n-3 PUFA (Serot et al., 2001), such as 2, 3-Pentanedione, which can give the muscle a sweet, butter-like and fruity smell, decreased with the increase of replacement level. And 3-Methylbutanal and 3-Methylbutanol, the products of degradation and oxidative deamination of Leucine $(\mathrm{Mu}$ et al., 2017), showed similar upward trend with Leucine content following the increase of TM meal inclusion. Previous study showed that the changes of fatty acid composition in fish fillet affect the total volatile compounds and thus affect the flavour of fish (Turchini et al., 2013). Therefore, the difference of muscle volatile compounds among treatments may be due to changes in muscle fatty acid and amino acid composition. Meanwhile, the fatty acid profile of fish muscle reflects the dietary fatty acid composition, which affects the quality and nutritional composition of fish muscle (Sánchez-Muros et al., 2014). In the present study, the inclusion of TM meal in diets affected the muscle fatty acid profile. The content of n-3 PUFA (such as EPA) significantly reduced, while the percentage of $n-6$ series PUFA, especially Linoleic acid (C18:2n-6) significantly increased with the increase of TM inclusion. Thus, a reduction of $\Sigma \mathrm{n}-3 / \Sigma \mathrm{n}-6$ PUFA ratio was detected following the increase of TM inclusion. Similar results were obtained in European sea bass (Gasco et al., 2016; Mastoraki et al., 2020), common catfish (Roncarati et al., 2015) and rainbow trout (Belforti et al., 2016). This is mainly because most terrestrial insects, including yellow mealworms, are notably deficient in EPA and DHA and rich in linoleic acid (Sánchez-Muros et al., 2014). The dietary fatty acid composition of the present study was determined, and the content of EPA and DHA decreased with the level of TM meal increasing. However, EPA and DHA have important biological functions in fish, but the rate of synthesis is lower than the nutritional requirement, so their addition in the feed is necessary.

\section{Conclusion}

In the present study, T. molitor meal can replace at least $30 \%$ of fishmeal protein in the diet for large yellow croaker without negative effects on the growth, feed utilisation and fish quality. The inclusion of TM meal in the diet improved the skin colour of large yellow croaker. In addition, from the perspective of body composition, texture and flavour analysis, histology and gene expression, higher replacement levels (75 and 100\%) can adversely affect the texture and flavour of fish fillets.

\section{Supplementary material}

Supplementary material can be found online at https://doi. org/10.3920/JIFF2021.0144.

Table S1. Composition of TASA fish meal.

\section{Acknowledgements}

This work was financially supported by the National Key R\&D Program of China (2019YFD0900200). 


\section{Conflict of interest}

The authors declare no conflict of interest.

\section{References}

Alasalvar, C., Taylor, K.D. and Shahidi, F., 2005. Comparison of volatiles of cultured and wild sea bream (Sparus aurata) during storage in ice by dynamic headspace analysis/gas chromatography-mass spectrometry. Journal of Agricultural \& Food Chemistry 53: 26162622. https://doi.org/10.1021/jf0483826

Association of Official Analytical Chemists (AOAC), 1995. Official methods of analysis, $16^{\text {th }}$ edition. AOAC, Arlington, VA, USA.

Association of Official Analytical Chemists AOAC, 2000. Official methods of analysis, $17^{\text {th }}$ edition. AOAC, Gaithersburg, MD, USA.

Ayala, M.D., López-Albors, O., Gil, F., Garca-Alcázar, A. and Moreno, F., 2001. Temperature effects on muscle growth in two populations (Atlantic and Mediterranean) of sea bass, Dicentrarchus labrax L. Aquaculture 202: 359-370. https://doi.org/10.1016/S00448486(01)00785-2

Belforti, M., Gai, F., Lussiana, C., Renna, M., Malfatto, V., Rotolo, L., De Marco, M., Dabbou, S., Schiavone, A., Zoccarato, I. and Gasco, L., 2016. Tenebrio molitor meal in rainbow trout (Oncorhynchus Mykiss) diets: effects on animal performance, nutrient digestibility and chemical composition of fillets. Italian Journal of Animal Science 14: 670-676. https://doi.org/10.4081/ijas.2015.4170

Bjørnevik, M., Hansen, H., Roth, B., Foss, A., Vikingstad, E., Solberg, C. and Imsland, A.K., 2017. Effects of starvation, subsequent feeding and photoperiod on flesh quality in farmed cod (Gadus morhua). Aquaculture Nutrition 23: 285-292. https://doi.org/10.1111/ anu.12391

Brown, M.L. and Murphy, B.R., 2004. Seasonal dynamics of direct and indirect condition indices in relation to energy allocation in largemouth bass Micropterus salmoides (Lacèpede). Ecology of Freshwater Fish 13: 23-26. https://doi.org/10.1111/j.09066691.2004.00031.x

Carter, C.G., He, Z.Y., Houlihan, D.F., McCarthy, I.D. and Davidson, I., 1995. Effect of feeding on the tissue free amino acid concentrations in rainbow trout (Oncorhynchus mykiss Walbaum). Fish Physiology and Biochemistry 14: 153-164. https://doi.org/10.1007/BF00002458

Commission International de I’ Eclairage (CIE), 1976. Official recommendations on uniform colour space, colour difference equations and metric colour terms. Suppl. No. 2 to CIE Publication No.15, Colorimetry. CIE, Paris, France.

Decking, U.K., Alves, C., Wallimann, T., Wyss, M. and Schrader, J., 2001. Functional aspects of creatine kinase isoenzymes in endothelial cells. American Journal of Physiology: Cell Physiology 281: C320-328. https://doi.org/10.1152/ajpcell.2001.281.1.C320

Dias, J., Huelvan, C., Dinis, M.T. and Metailler, R., 1998. Influence of dietary bulk agents (silica, cellulose and a natural zeolite) on protein digestibility, growth, feed intake and feed transit time in European seabass (Dicentrarchus labrax) juveniles. Aquatic Living Resources 11: 219-226. https://doi.org/10.1016/S0990-7440(98)89004-9
Feng, P., He, J., Lv, M., Huang, G., Chen, X., Yang, Q., Wang, J., Wang, D. and Ma, H., 2019. Effect of dietary Tenebrio molitor protein on growth performance and immunological parameters in Macrobrachium rosenbergii. Aquaculture 511: 734247. https:// doi.org/10.1016/j.aquaculture.2019.734247

Finke, M.D., 2002. Complete nutrient composition of commercially raised invertebrates used as food for insectivores. Zoo Biology 21: 269-285. https://doi.org/10.1002/zoo.10031

Finke, M.D., 2015. Complete nutrient content of four species of commercially available feeder insects fed enhanced diets during growth. Zoo Biology 34: 554-564. https://doi.org/10.1002/zoo.21246

Fuentes, A., Fernández-Segovia, I., Serra, J.A. and Barat, J.M., 2010. Comparison of wild and cultured sea bass (Dicentrarchus labrax) quality. Food Chemistry 119: 1514-1518. https://doi.org/10.1016/j. foodchem.2009.09.036

Fuke, S. and Konosu, S., 1991. Taste-active components in some foods - a review of Japanese research. Physiology \& Behavior 49: 863-868. https://doi.org/10.1016/0031-9384(91)90195-T

Gasco, L., Gai, F., Maricchiolo, G., Genovese, L., Ragonese, S., Bottari, T. and Caruso, G., 2018. Fishmeal alternative protein sources for aquaculture feeds, feeds for the aquaculture sector. In: Feeds for the aquaculture sector. SpringerBriefs in Molecular Science. Springer, Cham, Switzerland. https://doi.org/10.1007/978-3-319-77941-6_1

Gasco, L., Henry, M., Piccolo, G., Marono, S., Gai, F., Renna, M., Lussiana, C., Antonopoulou, E., Mola, P. and Chatzifotis, S., 2016. Tenebrio molitor meal in diets for European sea bass (Dicentrarchus labrax L.) juveniles: growth performance, whole body composition and in vivo apparent digestibility. Animal Feed Science and Technology 220: 34-45. https://doi.org/10.1016/j. anifeedsci.2016.07.003

Geering, K., 1990. Subunit assembly and functional maturation of Na,K-ATPase. Journal of Membrane Biology 115: 109-121. https:// doi.org/10.1007/BF01869450

Ginés, R., Valdimarsdottir, T., Sveinsdottir, K. and Thorarensen, H., 2004. Effects of rearing temperature and strain on sensory characteristics, texture, colour and fat of Arctic charr (Salvelinus alpinus). Food Quality and Preference 15: 177-185. https://doi. org/10.1016/s0950-3293(03)00056-9

Griffith, O. and Meister, A., 1980. Excretion of cysteine and gammaglutamylcysteine moieties in human and experimental animal gamma-glutamyl transpeptidase deficiency. Proceedings of the National Academy of Sciences 77: 3384-3387.

Grigorakis, K., 2007. Compositional and organoleptic quality of farmed and wild gilthead sea bream (Sparus aurata) and sea bass (Dicentrarchus labrax) and factors affecting it: a review. Aquaculture 272: 55-75. https://doi.org/10.1016/j.aquaculture.2007.04.062

Haard, N.F., 1992a. Biochemistry and chemistry of color and color change in seafoods. In: Flick Jr., G.J. and Martin, R.E. (eds.) Advances in seafood biochemistry. CRC Press, Boca Raton, FL, USA.

Haard, N.F., 1992b. Control of chemical-composition and food quality attributes of cultured fish. Food Research International 25: 289-307. https://doi.org/10.1016/0963-9969(92)90126-P

Hamada-Sato, N., Usui, K., Kobayashi, T., Imada, C. and Watanabe, E., 2005. Quality assurance of raw fish based on HACCP concept. Food Control 16: 301-307. https://doi.org/10.1016/j.foodcont.2004.02.001 
Hartman, K.J. and Margraf, F.J., 2006. Relationships among condition indices, feeding and growth of walleye in Lake Erie. Fisheries Management and Ecology 13: 121-130. https://doi.org/10.1111/ j.1365-2400.2006.00486.x

Hatae, K., Yoshimatsu, F. and Matsumoto, J.J., 1990. Role of musclefibers in contributing firmness of cooked fish. Journal of Food Science 55: 693-696. https://doi.org/10.1111/j.1365-2621.1990. tb05208.x

Henry, M., Gasco, L., Piccolo, G. and Fountoulaki, E., 2015. Review on the use of insects in the diet of farmed fish: past and future. Animal Feed Science and Technology 203: 1-22. https://doi.org/10.1016/j. anifeedsci.2015.03.001

Houlihan, D.F., Mathers, E.M. and Foster, A., 1993. Biochemical correlates of growth rate in fish. In: Rankin, J.C. and Jensen F.B. (eds.) Fish ecophysiology. Chapman \& Hall Fish and Fisheries Series. Vol. 9. Springer, Dordrecht, the Netherlands. https://doi. org/10.1007/978-94-011-2304-4_2

Hurling, R., Rodell, J.B. and Hunt, H.D., 1996. Fiber diameter and fish texture. Journal of Texture Studies 27: 679-685. https://doi. org/10.1111/j.1745-4603.1996.tb01001.x

Iaconisi, V., Marono, S., Parisi, G., Gasco, L., Genovese, L., Maricchiolo, G., Bovera, F. and Piccolo, G., 2017. Dietary inclusion of Tenebrio molitor larvae meal: effects on growth performance and final quality treats of blackspot sea bream (Pagellus bogaraveo). Aquaculture 476: 49-58. https://doi.org/10.1016/j.aquaculture.2017.04.007

Iaconisi, V., Secci, G., Sabatino, G., Piccolo, G., Gasco, L., Papini, A.M. and Parisi, G., 2019. Effect of mealworm (Tenebrio molitor L.) larvae meal on amino acid composition of gilthead sea bream (Sparus aurata L.) and rainbow trout (Oncorhynchus mykiss W.) fillets. Aquaculture 513: 734403. https://doi.org/10.1016/j. aquaculture.2019.734403

Johnston, I.A., Alderson, R., Sandham, C., Dingwall, A., Mitchell, D., Selkirk, C., Nickell, D., Baker, R., Robertson, B., Whyte, D. and Springate, J., 2000. Muscle fibre density in relation to the colour and texture of smoked Atlantic salmon (Salmo salar L.). Aquaculture 189: 335-349. https://doi.org/10.1016/S0044-8486(00)00373-2

Johnston, I.A., Li, X., Vieira, V.L.A., Nickell, D., Dingwall, A., Alderson, R., Campbell, P. and Bickerdike, R., 2006. Muscle and flesh quality traits in wild and farmed Atlantic salmon. Aquaculture 256: 323336. https://doi.org/10.1016/j.aquaculture.2006.02.048

Joulia, D., Bernardi, H., Garandel, V., Rabenoelina, F., Vernus, B. and Cabello, G., 2003. Mechanisms involved in the inhibition of myoblast proliferation and differentiation by myostatin. Experimental Cell Research 286: 263-275. https://doi.org/10.1016/s00144827(03)00074-0

Kaufman, S.D., Johnston, T.A., Leggett, W.C., Moles, M.D., Casselman, J.M. and Schulte-Hostedde, A.I., 2007. Relationships between body condition indices and proximate composition in adult walleyes. Transactions of the American Fisheries Society 136: 1566-1576. https://doi.org/10.1577/T06-262.1

Kikuchi, K., Sato, T., Furuta, T., Sakaguchi, I. and Deguchi, Y., 1997. Use of meat and bone meal as a protein source in the diet of juvenile Japanese flounder. Fisheries Science 63: 29-32. https:// doi.org/10.2331/fishsci.63.29
Kong, Y., Yang, X., Ding, Q., Zhang, Y.Y., Sun, B.G., Chen, H.T. and Sun, Y., 2017. Comparison of non-volatile umami components in chicken soup and chicken enzymatic hydrolysate. Food Research International 102: 559-566. https://doi.org/10.1016/j. foodres.2017.09.038

Konosu, S., 1979. The taste of fish and shellfish. In: Boudreau, J.C. (ed.) Food taste chemistry. American Chemical Society, Washington, DC, USA, pp. 185-203.

Kroeckel, S., Harjes, A.G.E., Roth, I., Katz, H., Wuertz, S., Susenbeth, A. and Schulz, C., 2012. When a turbot catches a fly: evaluation of a pre-pupae meal of the black soldier fly (Hermetia illucens) as fish meal substitute - growth performance and chitin degradation in juvenile turbot (Psetta maxima). Aquaculture 364-365: 345-352. https://doi.org/10.1016/j.aquaculture.2012.08.041

Liu, X., Han, B., Xu, J., Zhu, J., Hu, J., Wan, W. and Miao, S., 2020. Replacement of fishmeal with soybean meal affects the growth performance, digestive enzymes, intestinal microbiota and immunity of Carassius auratus gibelio $9 \times$ Cyprinus carpio $\widehat{\partial}$. Aquaculture Reports 18: 100472. https://doi.org/10.1016/j.aqrep.2020.100472

Loje, H., Jensen, K.N., Hyldig, G., Nielsen, H.H. and Nielsen, J., 2007. Changes in liquid-holding capacity, water distribution and microstructure during chill storage of smoked salmon. Journal of the Science of Food and Agriculture 87: 2684-2691. https://doi. org/10.1002/jsfa.3031

Longvah, T., Mangthya, K. and Ramulu, P., 2011. Nutrient composition and protein quality evaluation of eri silkworm (Samia ricinii) prepupae and pupae. Food Chemistry 128: 400-403. https://doi. org/10.1016/j.foodchem.2011.03.041

Lopez-Albors, O., Ayala, M.D., Gil, F., Garcia-Alcazar, A., Abellan, E., Latorre, R., Ramirez-Zarzosa, G. and Vazquez, J.M., 2003. Early temperature effects on muscle growth dynamics and histochemical profile of muscle fibres of sea bass Dicentrarchus labrax L., during larval and juvenile stages. Aquaculture 220: 385-406. https://doi. org/10.1016/S0044-8486(02)00532-X

Lv, H.B., Ma, Y.Y., Hu, C.T., Lin, Q.Y., Yue, J.J., Chen, L.Q., Zhang, M.L., Du, Z.Y. and Qiao, F., 2021. The individual and combined effects of hypoxia and high-fat diet feeding on nutrient composition and flesh quality in Nile tilapia (Oreochromis niloticus). Food Chemistry 343: 128479. https://doi.org/10.1016/j.foodchem.2020.128479

Ma, R., Meng, Y., Zhang, W. and Mai, K., 2019. Comparative study on the organoleptic quality of wild and farmed large yellow croaker Larimichthys crocea. Journal of Oceanology and Limnology 38: 260-274. https://doi.org/10.1007/s00343-019-8353-0

Makkar, H.P.S., Tran, G., Heuzé, V. and Ankers, P., 2014. Stateof-the-art on use of insects as animal feed. Animal Feed Science and Technology 197: 1-33. https://doi.org/10.1016/j. anifeedsci.2014.07.008

Mao, C., Hu, X. and Li, N., 2008. Identification and expression profile of a novel alternative splicing of $\operatorname{Pax} 7$ in chick skeletal muscle. Poultry Science 87: 1919-1925. https://doi.org/10.3382/ps.2007-00479

Martínez-Llorens, S., Moñino, A.V., Tomás Vidal, A., Salvador, V.J.M., Pla Torres, M. and Jover Cerdá, M., 2007. Soybean meal as a protein source in gilthead sea bream (Sparus aurata L.) diets: effects on growth and nutrient utilization. Aquaculture Research 38: 82-90. https://doi.org/10.1111/j.1365-2109.2006.01637.x 
Maruji, Y., Shimizu, M., Murata, M., Ando, M., Sakaguchi, M. and Hirata, T., 2010. Multiple taste functions of the umami substances in muscle extracts of yellowtail and bastard halibut. Fisheries Science 76: 521-528. https://doi.org/10.1007/s12562-010-0231-9

Mastoraki, M., Mollá Ferrándiz, P., Vardali, S.C., Kontodimas, D.C., Kotzamanis, Y.P., Gasco, L., Chatzifotis, S. and Antonopoulou, E., 2020. A comparative study on the effect of fish meal substitution with three different insect meals on growth, body composition and metabolism of European sea bass (Dicentrarchus labrax L.). Aquaculture 528: 735511. https://doi.org/10.1016/j. aquaculture.2020.735511

Merlo, T.C., Lorenzo, J.M., Saldana, E., Patinho, I., Oliveira, A.C., Menegali, B.S., Selani, M.M., Dominguez, R. and Contreras-Castillo, C.J., 2021. Relationship between volatile organic compounds, free amino acids, and sensory profile of smoked bacon. Meat Science 181: 108596. https://doi.org/10.1016/j.meatsci.2021.108596

Mu, H., Wei, Z., Yi, L., Liang, H., Zhao, L., Zhang, W. and Mai, K., 2017. Dietary fishmeal levels affect the volatile compounds in cooked muscle of farmed large yellow croaker Larimichthys crocea. Aquaculture Research 48: 5821-5834. https://doi.org/10.1111/ are. 13405

Ng, W.K., Liew, F.L., Ang, L.P. and Wong, K.W., 2001. Potential of mealworm (Tenebrio molitor) as an alternative protein source in practical diets for African catfish, Clarias gariepinus. Aquaculture Research 32: 273-280. https://doi.org/10.1046/j.1355557x.2001.00024.x

Ninomiya, Y., 1987. Qualitative discrimination among 'umami' and the four basic taste substances in mice. In: Kawamura, Y. and Morley, R.K. (eds.) Umami: a basic taste. Marcel Dekker Inc., New York, NY, USA.

Nogales-Mérida, S., Gobbi, P., Józefiak, D., Mazurkiewicz, J., Dudek, K., Rawski, M., Kierończyk, B. and Józefiak, A., 2018. Insect meals in fish nutrition. Reviews in Aquaculture 11: 1080-1103. https:// doi.org/10.1111/raq.12281

Ocaño-Higuera, V.M., Maeda-Martínez, A.N., Marquez-Ríos, E., Canizales-Rodríguez, D.F., Castillo-Yáñez, F.J., Ruíz-Bustos, E., Graciano-Verdugo, A.Z. and Plascencia-Jatomea, M., 2011. Freshness assessment of ray fish stored in ice by biochemical, chemical and physical methods. Food Chemistry 125: 49-54. https:// doi.org/10.1016/j.foodchem.2010.08.034

Østbye, T.K.K., Ruyter, B., Standal, I.B., Stien, L.H., Bahuaud, D., Dessen, J.E., Latif, M.S., Fyhn-Terjesen, B., Rørvik, K.A. and Mørkøre, T., 2018. Functional amino acids stimulate muscle development and improve fillet texture of Atlantic salmon. Aquaculture Nutrition 24: 14-26. https://doi.org/10.1111/anu.12528

Pangle, K.L. and Sutton, T.M., 2010. Temporal changes in the relationship between condition indices and proximate composition of juvenile Coregonus artedi. Journal of Fish Biology 66: 1060-1072. https://doi.org/10.1111/j.0022-1112.2005.00660.x

Pavlidis, M., Papandroulakis, N. and Divanach, P., 2006. A method for the comparison of chromaticity parameters in fish skin: preliminary results for coloration pattern of red skin Sparidae. Aquaculture 258: 211-219. https://doi.org/10.1016/j.aquaculture.2006.05.028
Periago, M.J., Ayala, M.D., López-Albors, O., Abdel, I., Martínez, C., García-Alcázar, A., Ros, G. and Gil, F., 2005. Muscle cellularity and flesh quality of wild and farmed sea bass, Dicentrarchus labrax L. Aquaculture 249: 175-188. https://doi.org/10.1016/j. aquaculture.2005.02.047

Piccolo, G., Iaconisi, V., Marono, S., Gasco, L., Loponte, R., Nizza, S., Bovera, F. and Parisi, G., 2017. Effect of Tenebrio molitor larvae meal on growth performance, in vivo nutrients digestibility, somatic and marketable indexes of gilthead sea bream (Sparus aurata). Animal Feed Science and Technology 226: 12-20. https://doi.org/10.1016/j. anifeedsci.2017.02.007

Richter, B.L., Silva, T.S.D., Michelato, M., Marinho, M.T., Goncalves, G.S. and Furuya, W.M., 2021. Combination of lysine and histidine improves growth performance, expression of muscle growthrelated genes and fillet quality of grow-out Nile tilapia. Aquaculture Nutrition 27: 568-580. https://doi.org/10.1111/anu.13207

Robinson, E.H. and Li, M.H., 1994. Use of plant proteins in catfish feeds: replacement of soybean meal with cottonseed meal and replacement of fish meal with soybean meal and cottonseed meal. Journal of the World Aquaculture Society 25: 271-276. https://doi. org/10.1111/j.1749-7345.1994.tb00190.x

Roncarati, A., Gasco, L., Parisi, G. and Terova, G., 2015. Growth performance of common catfish (Ameiurus melas Raf.) fingerlings fed mealworm (Tenebrio molitor) diet. Journal of Insects as Food and Feed 1: 233-240. https://doi.org/10.3920/jiff2014.0006

Saito, T., Arai, K.I. and Matsuyoshi, M., 1959. A new method for estimating the freshness of fish. Bulletin of the Japanese Society of Scientific Fisheries 24: 749-750.

Sánchez-Alonso, I., Haji-Maleki, R. and Borderias, A.J., 2007. Wheat fiber as a functional ingredient in restructured fish products. Food Chemistry 100: 1037-1043. https://doi.org/10.1016/j. foodchem.2005.09.090

Sánchez-Muros, M.-J., Barroso, F.G. and Manzano-Agugliaro, F., 2014. Insect meal as renewable source of food for animal feeding: a review. Journal of Cleaner Production 65: 16-27. https://doi.org/10.1016/j. jclepro.2013.11.068

Sato, K., Yoshinaka, R., Sato, M. and Shimizu, Y., 1986. Collagen content in the muscle of fishes in association with their swimming movement and meat texture. Nippon Suisan Gakkaishi 52: 15951600. https://doi.org/10.2331/suisan.52.1595

Schabel, H.G., Durst, P.B., Johnson, D.V., Leslie, R.N. and Shono, K., 2010. Forest insects as food: a global review. In: Durst, P.B., Johnson, D.V., Leslie, R.N. and Shono, K. (eds.) Forest insects as food: humans bite back. Proceedings of a workshop on Asia-Pacific resources and their potential for development. 19-21 February 2008. Chiang Mai, Thailand

Serot, T., Regost, C., Carole, P., Jean, R. and Jacqueline, A., 2001. Effect of dietary lipid sources on odour-active compounds in muscles of turbot (Psetta Maxima). Journal of the Science of Food and Agriculture 81: 1339-1346. https://doi.org/10.1002/jsfa.950

Sigholt, T., Erikson, U., Rustad, T., Johansen, S., Nordtvedt, T.S. and Seland, A., 1997. Handling stress and storage temperature affect meat quality of farmed-raised Atlantic salmon (Salmo salar). Journal of Food Science 62: 898-905. https://doi.org/10.1111/j.1365-2621.1997. tb15482.x 
Stamer, A., 2015. Insect proteins-a new source for animal feed: the use of insect larvae to recycle food waste in high-quality protein for livestock and aquaculture feeds is held back largely owing to regulatory hurdles. EMBO Reports 16: 676-680. https://doi. org/10.15252/embr.201540528

Su, J., Gong, Y., Cao, S., Lu, F., Han, D., Liu, H., Jin, J., Yang, Y., Zhu, X. and Xie, S., 2017. Effects of dietary Tenebrio molitor meal on the growth performance, immune response and disease resistance of yellow catfish (Pelteobagrus fulvidraco). Fish \& Shellfish Immunology 69: 59-66. https://doi.org/10.1016/j.fsi.2017.08.008

Sun, H., Tang, J.-w., Yao, X.-h., Wu, Y.-f., Wang, X., Liu, Y. and Lou, B., 2015. Partial substitution of fish meal with fermented cottonseed meal in juvenile black sea bream (Acanthopagrus schlegelii) diets. Aquaculture 446: 30-36. https://doi.org/10.1016/j. aquaculture.2015.04.020

Tocher, D.R., 2003. Metabolism and functions of lipids and fatty acids in teleost fish. Reviews in Fisheries Science 11: 107-184. https:// doi.org/10.1080/713610925

Turchini, G.M., Hermon, K., Moretti, V.M., Caprino, F., Busetto, M.L., Bellagamba, F., Rankin, T. and Francis, D.S., 2013. Seven fish oil substitutes over a rainbow trout grow-out cycle: II) effects on final eating quality and a tentative estimation of feed-related production costs. Aquaculture Nutrition 19: 95-109. https://doi. org/10.1111/anu.12045

Van Huis, A. and Dunkel, F.V., 2017. Edible insects: a neglected and promising food source. In: Nadathur, S.R., Wanasundara, J.P.D. and Scanlin, L. (eds.) Sustainable protein sources. Academic Press, Cambridge, MA, USA, pp. 341-355. https://doi.org/10.1016/b9780-12-802778-3.00021-4

Van Huis, A., 2013. Potential of insects as food and feed in assuring food security. Annual Review of Entomology 58: 563-583. https:// doi.org/10.1146/annurev-ento-120811-153704

Van Huis, A., 2020. Insects as food and feed, a new emerging agricultural sector: a review. Journal of Insects as Food and Feed 6: 27-44. https://doi.org/10.3920/jiff2019.0017

Veggetti, A., Mascarello, F., Scapolo, P.A. and Rowlerson, A., 1990. Hyperplastic and hypertrophic growth of lateral muscle in Dicentrarchus labrax (L.). Anatomy and Embryology 182: 1-10.

Vélez, E.J., Lutfi, E., Azizi, S., Perelló, M., Salmerón, C., Riera-Codina, M., Ibarz, A., Fernández-Borràs, J., Blasco, J., Capilla, E., Navarro, I. and Gutiérrez, J., 2017. Understanding fish muscle growth regulation to optimize aquaculture production. Aquaculture 467: 28-40. https:// doi.org/10.1016/j.aquaculture.2016.07.004

Villanueva, J., Vanacore, R., Goicoechea, O. and Amthauer, R., 1997. Intestinal alkaline phosphatase of the fish Cyprinus carpio: regional distribution and membrane association. Journal of Experimental Zoology 279: 347-355. https://doi.org/10.1002/(Sici)1097010x(19971101)279:4<347::Aid-Jez4>3.0.Co;2-O
Wang, B., Liu, Y., Feng, L., Jiang, W.D., Kuang, S.Y., Jiang, J., Li, S.H., Tang, L. and Zhou, X.Q., 2015. Effects of dietary arginine supplementation on growth performance, flesh quality, muscle antioxidant capacity and antioxidant-related signalling molecule expression in young grass carp (Ctenopharyngodon idella). Food Chemistry 167: 91-99. https://doi.org/10.1016/j. foodchem.2014.06.091

Watson, A.M., Buentello, A. and Place, A.R., 2014. Partial replacement of fishmeal, poultry by-product meal and soy protein concentrate with two non-genetically modified soybean cultivars in diets for juvenile cobia, Rachycentron canadum. Aquaculture 434: 129-136. https://doi.org/10.1016/j.aquaculture.2014.08.003

Wei, Y., Chen, H., Jia, M., Zhou, H., Zhang, Y., Xu, W., Zhang, W. and Mai, K., 2019a. Effects of dietary Antarctic krill Euphausia superba meal on growth performance and muscle quality of triploid rainbow trout Oncorhynchus mykiss farmed in sea water. Aquaculture 509: 72-84. https://doi.org/10.1016/j.aquaculture.2019.05.013

Wei, Y., Shen, H., Xu, W., Pan, Y., Chen, J., Zhang, W. and Mai, K., 2019b. Replacement of dietary fishmeal by Antarctic krill meal on growth performance, intestinal morphology, body composition and organoleptic quality of large yellow croaker Larimichthys crocea. Aquaculture 512: 734281. https://doi.org/10.1016/j. aquaculture.2019.734281

Wei, Z., Ma, J., Pan, X., Mu, H., Li, J., Shentu, J., Zhang, W. and Mai, K., 2016. Dietary hydroxyproline improves the growth and muscle quality of large yellow croaker Larimichthys crocea. Aquaculture 464: 497-504. https://doi.org/10.1016/j.aquaculture.2016.07.015

Weintraub, H., 1993. The MyoD family and myogenesis: redundancy, networks, and thresholds. Cell 75: 1241-1244. https://doi. org/10.1016/0092-8674(93)90610-3

Xu, G.F., Wang, Y.Y., Han, Y., Liu, Y., Yang, Y.H., Yu, S.L. and Mou, Z.B., 2015. Growth, feed utilization and body composition of juvenile Manchurian trout, Brachymystax lenok (Pallas) fed different dietary protein and lipid levels. Aquaculture Nutrition 21: 332-340. https:// doi.org/10.1111/anu.12165

Yamaguchi, S. and Ninomiya, K., 2000. Umami and food palatability. Journal of Nutrition 130: 921S-926S. https://doi.org/10.1093/ jn/130.4.921S

Yi, X., Li, J., Xu, W., Zhang, W. and Mai, K., 2016. Effects of dietary lutein/canthaxanthin ratio on the growth and pigmentation of large yellow croaker Larimichthys croceus. Aquaculture Nutrition 22: 683-690. https://doi.org/10.1111/anu.12289

Yi, X., Xu, W., Zhou, H., Zhang, Y., Luo, Y., Zhang, W. and Mai, K., 2014. Effects of dietary astaxanthin and xanthophylls on the growth and skin pigmentation of large yellow croaker Larimichthys croceus. Aquaculture 433: 377-383. https://doi.org/10.1016/j. aquaculture.2014.06.038

Zhu, W., Luan, H., Bu, Y., Li, J., Li, X. and Zhang, Y., 2021. Changes in taste substances during fermentation of fish sauce and the correlation with protease activity. Food Research International 144: 110349. https://doi.org/10.1016/j.foodres.2021.110349 